Aus dem Veterinär-anatomischen Institut der Universität Zürich

(Prof. Dr. Zietzschmann).

\title{
Der muskulöse Apparat der Iris des Schafes and seine Beziehungen zur Gestalt der Pupille.
}

\author{
Von \\ Hans Richter, \\ Prosektor.
}

Mit Taf. IX u. X, Fig, 1-11.

In der kürzlich erschienenen Arbeit „Die inneren Trisschichten der Haussäugetiere" berichtet Klinge (8), dass er bei Rind, Schaf, Ziege und Schwein Verstärkungen des Dilatator iridis durch Bildung von Einfaltungen und Fortsätzen der äusseren Retinalamelle gefunden hat, ähnlich wie in stark ausgeprägtem Masse Heerfordt sie bei dem Seehund gefunden hat und Grynfeltt sie bei dem Delphin sah. Klinge gibt eine genaue Beschreibung des Aufbaues dieser Bildung bei den erwähnten Haustieren, sowie gewisser Veränderungen, die bei den verschiedenen Pupillenstellungen an ihnen nachweisbar sind. Er macht auch einige Angaben über den Verlauf dieser Verstärkungen, die sich als Leisten präsentieren, in der Richtung vom Ciliarkörper nach dem Pupillarrande zu. Diese Angaben können aber nicht den Anspruch auf Vollständigkeit machen, da dieselben nicht durch Studien an durchgehenden Serien gewonnen sind. Eine weitere Lücke in den Untersuchungen ergibt sich daraus, dass Klinge nirgends Andeutungen darüber macht, aus welchen Teilen des ganzen Irisringes er sein Untersuchungsmaterial genommen hat. Es fehlen also gänzlich Angaben darüber, wie die Verstärkungsbildungen des Dilatators in den einzelnen Abschnitten des Irisringes auftreten. Diese Seite der Frage scheint um so mehr einer Berücksichtigung wert, als wir es bei Rind, Schaf und Ziege mit einer ausgesprochen querovalen Pupille zu tun haben. Bei dem Pupillenspiel sind bei dieser Gestaltung der Iris hauptsächlich nur die an den Langseiten des pupillaren Querovales gelegenen Teile des Irisringes v. Graefe's Archiv für Ophthalmologie. IXX. 3. 
beteiligt. Man muss annehmen, dass sich das funktionelle Verhalten im anatomischen Aufbau widerspiegelt. Fasst man nun mit Klinge und andern Autoren die Einfaltungen der Dilatatorzellschicht als Verstärkungsbildung auf, so liegt die Vermutung nahe, dass im Gegensatz zu den Schmalseiten die Einfaltungen an den Langseiten stärker ausgeprägt sein werden. Aus diesen Erwägungen heraus und in der Absicht, in diesen Punkten die Klingesche Arbeit zu vervollständigen und zu ergänzen, sind vorliegende Untersuchungen unternommen worden. Ich beschränkte mich hierbei auf die Iris nur einer Tiergattung, nämlich des Schafes, um nach recht vielen Seiten hin die Frage möglichst eingehend prüfen zu können.

Bezäglich der Literatur utber den Dilatator bei Säugern im allgemeinen und den Leisten des Dilatators im speziellen, kann ich in der Hauptsache auf die Ansführungen in der Arbeit von Klinge(8) verweisen. Ich kann es mir jedoch nicht versagen, gewisse Angaben einzelner Autoren, die in engerem Zusammenhang mit meinen Untersuchungen stehen, eingehender wiederzugeben.

Heerfordt $(7)$ behandelt die radiäre Faltenbildung an der Innenfläche $\left.{ }^{1}\right\rangle$ der Iris und ihre Bedeutung als Verstärkung des Dilatators am eingehendsten und kritischsten. Indem or die Kontraktionsfalten als temporäre Bildungen aus dem Bereich der Betrachtung lässt, unterseheidet er zwei Formen radiärer Faltenbildung an der Innenfläche der Iris: 1. Radiäre Strukturfalten (Sehwalbe): Falten- und Furchenbildungen an der Innenfläche der Iris, an der sich die beiden hinteren Irisschichten (das sind Bruchsche Membran und änssere Pigmentzellsehicht, und innere Pigmentsehicht) beteiligen; 2. Dilatatorleisten; das sind Einfaltungen in das Irisstroma, die nur von der Dilatatorlamelle (Bruehsche Membran und äussere Pigmentzellschicht) gebildet werden.

[Beim Kaninchen beschreibt Heerfordt noeh eine andere Art von Faltenbildung, die ich im Gegensatz zu den beiden oben ervähnten Bildungen, die als Einfaltung (in das Stroma hinein) zu denten sind, als A us faltung bezeichnen möchte. Es sind jene hohen radiären Kämme, die der Innenfläche der Iris aufsitzen und ciliarwärts, dadurch dass sie mit der Vorderfläche der Ciliarfortsätze verschmelzen, Anlass geben zu einer Nischenbildung in dem peripheren Teil der hinteren Augenkammer, also in dem Raum zwischen den Ciliarfortsätzen und der Irisinnenfläche. Diese Falten haben gleichfalls einen Überzug, der von den beiden inneren Irisschichten gebildet wird. Die Dilatatorsehicht ist aber hier nur unvollkommen in Muskelzellen umgebildet; infolgedessen kommen diese Falten als Ver-

1) Bezüglich der" Lagebezeichnungen halte ich mich an die Ausführungen Zietzschmanns (11)im Handbuch der vergleichenden mikroskopischen Anatomie der Haustiere von Ellenberger. Innen bzw. aussen heisst demnach dem Bulbuszentrum näher, bzw. weiter von ihm entfernt gelegen. 
stärkung des Dilatators kaum in Betracht. Heerfordt elwähnt ihr Vorkommen nur beim Kaninehen.]

Die radiären Strukturfalten, die erste Form der Verstärkung des Dilatators sind in ihrem Auftreten an das Vorhandensein der Bruchschen Membran gebunden. Sie beginnen als sehr niedrige hinter dem Sphinkter gerade an dem Orte, wo zugleich die Umbildung der vorderen Epithelschicht in Muskelzellen beginnt. Ausserdem entwickeln sich die radiären Strukturfalten gerade zu derselben Zeit, da die vordere Epithelschicht des Muse. dilatator pupillae umgebildet wird. Ihr Vorkommen erwähnt Heerfordt bei Mensch, Kaninchen und Kalb und meint, dass sie wohl bei allen höheren Wirbeltieren zu konstatieren sind.

Die zweite Form der Verstärkung des Dilatators ist die Bildung von echten Dilatatórfalten oder Dilatatorleisten. Diese werden allein durch die äussere Epithelsehicht gebildet, ohne Beteiligung der inneren Pigmentschicht. Heerfordt stellt sie in Analogie mit den Einfaltungen der epithelialen Muskeln in das Bindegewebe bei den Aktinien. Besonders stark ausgeprägt hat er diese Leisten in der Iris des Seehundes gefunden. Doch sagt er: "Nach dem Studium der Iris des Seehundes fühlt man sich überzengt, dass man auch in der Iris des Menschen und des Kaninchens ganz kleine Andentungen echter Einfaltung aus der Tiefe der radiären Irisfalten am deutlichsten in der Nähe der Proc. ciliares gesehen hat." Beim Kalbe erwähnt Heerfordt das Vorkommen der echten Dilatatorfalten nicht, da er dieses Tier nicht zum Gegenstand genauerer: Untersuchungen nach dieser Richtung hin gemacht hat. Er gibt jedoch von einem Tangentialschnitt eine Zeichnung, allerdings nur bei sehwacher Vergrösserung gesehen. Diese lässt unsehwer den Schluss $\mathrm{zn}$, dass sich auch hier Dilatatorleisten vorfinden. Bei der Fischotter vermutet Heerfordt eine ähnliche Ausbildung der Dilatatorfalten wie beim Seehund, da Grünhagen(4) auf die gewaltige Entwicklung des Dilatators bei diesem Tiere zugleich mit der beim Seehunde hingewiesen hat.

Grynfeltt(5), der seine Untersuehungen auf zahlreiche Tiergattungen ansgedehnt hat, erwähnt anch die beiden Formen der Einfaltungen der Irisinnenfläche: 1. die radiären Strukturfalten, 2. die echten Dilatatorfalten, die er "plis musculaires" nennt. Ausserdem spricht er von Unebenheiten und Auszackungen, die die Bruchsehe Membran häufig nach dem Stroma hin zeigt. Ausserordentlich zahlreich fand Grynfeltt die Plis. muse. in der Iris des Delphins. Die Einfaltungen stehen hier so dicht nebeneinander, dass nur bei starker Vergrösserung die schmalen Zwischenräume des Stromas und dünne Gefässe zwisehen den einzelnen Plis zu erkennen sind. Bei den Haussäugetieren, die Grynfeltt untersucht hat, fand or Plis musculaires radiés bei Kaninchen, Meerschweinchen, Hund und Schaf. Ihr Vorkommen erwähnt er nicht bei Rind, Ziege und Schwein, deren Iris er auch zum Gegenstand seiner Untersuchungen gemacht hat. Das Verhalten beim Schaf beschreibt er genaner und gibt an, dass bei diesem Tiere mehr oder weniger tief einschneidende Strukturfalten vorhanden sind, von deren Seiten der Dilatator plis musculaires, die manchmal verzweigt sein können, aussendet.

Nach Klinge (8) kommen ausser den gewöhnlichen Radiärfalten (Struk- 
turfalten), wie sie alle von ihm untersuchten Tiere an der Irisinnenfläche zeigen, Dilatatorleisten bei Rind, Schaf, Ziege und Schwein vor.

Beim Schwein sind die Dilatatorleisten am kleinsten und nur bei Mydriasis vorhanden. Die einzelnen Elemente sind nicht in typischer: Faltenform geordnet, nåmlỉch so, dass das Protoplasma mit Kern und Pigment nach innen gelagert ist, während der kontraktile Teil in der Peripherie der Faltung liegt. Vielmehr sind die Zellen bunt durcheinander gelagert. Bei Miosis versehwinden die Leisten ganz.

Starker entwickelt, aber anch mit derselben Durcheinanderlagerung bei weiter Pupille finden sich die Dilatatorleisten bei Schaf und Ziege. Bei Miosis sollen sie sich jedoch grossenteils in ganz oder halb geschlossene Röhren mit typischer Faltenlagerung der einzelnen Dilatatorzellen umwandeln,

Beim Rinde fand Klinge ausser stark ausgebildeten Strukturfalten schon bei Mydriasis typische faltenartige Leisten. Bei Miosis sollen diese Leisten mehr oder weniger vollständig verschwinden.

Bezinglich des radiären Verlanfes der Leisten sagt Klinge, dass sie pupillenwărts in den Sphinkter auslaufen und als modifizierte Radiărfasern (Speichenfasern) aufgefasst werden können.

\section{Eigene Untersuohungen.}

Untersuchungsmaterial and Technik.

Die Schafaugen, die mir das Material zu meinen Untersuchungen lieferten, wurden stets lebenswarm in $10 \%$ Formalin- $(4 \%$ Formaldehyd-)alkohol $(30 \%)$ fixiert. Damit die Fixationsflüssigkeit schneller auf die Iris einwirken konnte, erölinhete ich vowher das Auge meist durch einen Schnitt in der Aquatorgegend des Bulbus und entfernte die Linse. Häufig schnitt ich auch die Cornea an, um so durch die eröffnete vordere Augenkammer die Fixationsflüssigkeit auf die Iris direkt einwirken zu lassen. Ieh erhielt so stets gut fixierte Präparate. Zur Erreichung der Miosis eserinisierte ich intra vitam die Augen und fixierte durch schnelles Einlegen in Formalinalkohol nach vorheriger Eröffnung der vorderen Augenkammer. Da es mir darauf ankam, beim Vergleieh der miotischen mit der mydriatischen Iris genau zu gehen and anch etwaige individuelle Verschiedenheiten auszuschliessen, so suchte ich von demselben Schafe das eine Auge mit weiter, das andere mit enger. Pupille za erhalten. Za diesem Zwecke eserinisierte ich das eine Auge etwa eine bis eine halbe Stunde vor der Schlachtung, indem ich offter Physostigminlösung einträufelte. Nach der Tötung, die durch betäubenden Keulensehlag und Verblutenlassen durch Halsschnitt wie üblich erfolgte, schnitt ich die Augen schnell heraus and fixicrte in oben angegebener Weise. Ich erlangte so von ein und demselben Individuum zwei Augen mit gut ausgeprägter entgegengesetzter Pupillenstellung. Jeden Irisring, den ich zur weiteren mikroskopischen Untersuchnng benutzte, photographierte (oder zeichnete) ich in natürlicher Grösse ab. So war einmal die Forro und Grösse des Irisringes und der Pupille festgelegt, sodann vermochte ich in dem Bilde mit genügender Genaugkeit die Lage der Schnitte einzuzeichnen, durch die ich den 
Irisring zur Weiterbehandlung und mikroskopischen Untersuchung zerlegte. Einzelne Irisringe bettete ich auch im ganzen in ihrem natürlichen $\mathrm{Zu}$ sammenhang mit Sklera und Cornea in Celloidin ein. Im übrigen verwandte ich zur Einbettung je nach Bedürfnis sowohl Paraffin als auch Celloidin. Die Methode war die bei diesen Formen übliche. Bei Paraffin sah ich darauf, die Dauer der Einbettung möglichst abzukürzen. Das lange Verweilen in dem Lösungsmittel des Paraffins wie auch in Thermostaten beeinträchtigt die Schnittfähigkeit des Irisgewebes ausserordentlich. Die einzelnen Teile des Irisringes zerlegte ich auf diese Weise in Serien von Tangential-, Radiär- und Flachschnitte. Die Schnittdicke schwankte bei Paraffinschnitten zwischen 5 und $12 \mu$, bei den Celloidinschnitten zwischen 10, 30 und $50 \mu$, je nach Zweck. Um Schnitte mit und ohne Pigment zum Vergleich nebeneinander zu haben, nahm ich nach einer bestimmten Anzahl von Schnitten einen oder mehrere beim Auflegen aus der Serie heraus, und unterzog sie der Depigmentation. Ich wandte dieselbe Methode wie Klinge an, die von Alex. Alfieri (1). Dieselbe besteht in der Vorbehandlung mit schwacher Kaliumpermanganatlösung und Nachbehandlung mit Oxalsäure. Ich versuchte auch die Methode von Grynfeltt und Mestresat (6), jedoch erhielt ich nicht so gute Resultate damit wie mit der ersten Methode. Die Tinktionsfähigkeit erseheint mir bei der letzten $\mathrm{Me}$ thode stärker beeinträchtigt zu sein. Die Befestigung der in Paraffinbändern vorliegenden Serien, aus den kleinen Schnitten von überwiegend bindegewebiger Natur bestehend, bot besondere Schwierigkeit, zumal wenn depigmentiert werden sollte. Ich gelangte zum Ziel mit einer Methode, die zuerst von Apáthy empfohlen ist (2). Ich tanchte die Objektträger mit den in der üblichen Weise mit Wasser aufgeklebten und getrockneten Paraffinschnitten in eine dünne (ungefähr $2 \%$ ) Celloidinlösung und liess rasch den Überschuss ablaufen. Dann steilte ich die Objektträger zum Trocknen auf. Man erhäIt auf diese Weise einen hautartigen Überzug von Celloidin. Zur besseren Befestigung desselben umrandete ich vorher das Präparatenfeld mit Eiweissglycerin oder mit Gelatine, die ich durch Eintanchen in Formalinlösung wie bei der Oltschen Aufklebemethode (10) unlöslich gemacht. Zur Befestigung der Celloidinserien auf den Objektträgern wandte ich mit Vorteil die Oltsche Aufklebemethode (10) an. Zur Färbung wandte ich in der Hauptsache Hämatoxylin oder Hämalaun, bei pigmenthaltigen Schnitten Nachfärbung in Eosin, van Gieson und Eisenhämatoxylin nach Heidenhain an.

Physiologische Vorbemerkungen.

Bevor ich die feineren mikroskopischen Verhältnisse im Irisringe des Schafes darlege, muss ich auf die gröbere Anatomie und einige damit zusammenhängende physiologische Fragen näher eingehen, soweit sie für meine Untersuchungen wichtig sind. Iris und Ciliarkörper hängen anatomisch eng miteinander zusammen. Das Corpus ciliare setzt sich zusammen aus dem Orbiculus ciliaris, dem Ciliarmuskel und der weiter corneawärts gelegenen Ciliarkrone. Die Corona ciliaris besteht wieder aus eimem Hauptteil, nämlich der Grundplatte und ihren Anbängen, den Ciliarfortsätzen; die Proc. ciliares strahlen von der ganzen inneren Fläche der ringförmigen 
Grundplatte bis zu lhrem corneaseitigen Rande hin ab und streben, frei aber diesen Rand vorspringend und etwas nach innen abgeneigt, dem Linsenäquator zu. Der gleiche corneaseitige Rand der Gruadplatte setzt sich in die Iris fort; diese zieht gleichsam als Tangente an die Vorderfläche der Linse. Im Meridionalschnitt (Taf. X, Fig. 2-9) betrachtet, trägt also der corneaseitige Rand der" Grundplatte zwei ungleichlange Gabeläste, die einen spitzen Winkel miteinander bilden. Der äussere längere Ast ist die Iris, der innere kürzere Ast wird vou den über den Rand vorspringenden Teilen der Proc. cil. gebildet.

Physiologisch und funktionell aufgefasst stellt die Iris den Blendapparat für die Augencamera dar. Vom physiologischen Standpunkte aus müssen wir daher die Iris (d. i. die funktionelle Iris) soweit begrenzen, als sie sich an der Vergrösserung und Verkleinewng der Pupille direkt beteiligt. Die Ciliarfortsätze mit den ron ihnon ausgehenden Faden der Zonula Zinnii sind ein Teil des Accommodationsapparates der Linse. Die Accommodation der Linse und das Pupillenspiel sind aber zwei Funktionen, die unabhängig voneinander ansgeübt werden und ausgetibt werden müssen. Wie wir nun oben sahen, stehen Iris und Ciliarfortsätze anatomisch in direktem Zusammenhange. Weiterhin ist von vielen Seiten nachgewiesen, dass der Dilatator peripher nur bis zum Scheitel des Irisciliarfortsatzwinkels reicht. In dem von dieser Stelle peripher gelegenen Teile der Iris sind keine Einrichtungen vorhanden, die diesen Teil in seiner Radiärbreite verringern könnten. Aber auch eine Dehnumg dieses Teiles infolge Kontraktion des Sphincter iridis kann nicht erfolgen. Geschähe dies, so würden bei Miosis glejehzeitig die Fäden der Zonula Zinnii, die darch die Processus ciliares mit diesem peripheren Teil der Iris in Verbindung stehen, entspann werden, was eine Accommodationsänderung der Linse bedenten würde. Es ist aber eine erwiesene Tatsache, dass Pupillenspiel und Accommodation vollkommen unabhängig voneinander vor sich gehen. Wenn wir also die Grenze der ,funktionellen" Iris festlegen wollen, so mütsen wir sagen, dass dieselbe im Scheitel des Winkels za suchen ist, der von der lris und den uiberragenden Teilen der Ciliarfortażtue gebildet wird. Ton dieser Stelle an bis zum Pupillarrande ist die. Iris fiu sich dehnungs- und kontraktiongfuhig. Wenn wir uns an einem Schafauge nach volliger Freilegung der vorderen Augenkammer durch Entfernung der Cornea und der tiberragenden Teile der Sklera die Iris betrachten, so sehen wir, dass thre periphere Umrandung hier ein breites eiförmiges Queroval bildet, dessen stampferer Teil nasal liegt (vgl. hierzu und fur die tolgenden Ansfuhrungen Taf. IX, Fig. 1). Der Iris mit dieser Unagrenzung möchte ich die Bezeichnung „Scheinoder Ausseniris" geben. Anders erseheint die Iris, wenn wir sie nach Eritfernung der Linse von junen betuchter. Wir sehen dann pon der Seheininis nur einen etwa kreisformigen Ausschnit, dessen periphere Unarandung von den zentralwärts überragenden Spitzen der einen Kranz bildenden Ciliarfortsătze gebildet wird. Nun kann man sich durch Padiarschnitte uberzeugen, dass der Tell der Irisfortsatze, der uber den Seheitelpunkt des Ciliarfortsatziniswinkels zentralwärts überragt, in allen Teilen des Irisinges angefäh gleich lang ist; demnach hat auch die Grenzlinie der "tunktionellen" Iris, die durch die Scheitelpunkte des Iriseiliarfortsatawinkels gelit, 
Kreisform im Gegensatz zur eiförmigen Umrandung der Aussen- oder Scheiniris. $Z u$ demselben Resultat kommt man anch, wenn man die überragenden Teile des Ciliarfortsatzkranzes bis zu dem Scheitel des Irisciliarfortsatzwinkels mit der Schere abträgt. Die Anpassung des Ciliarfortsatzkranzes an die kreisförmige Umrandung der Linse ist also nicht dadurch erreicht, dass der frei zentralwärts vorspringende Teil der Irisfortsätze in einzelnen Partien des Irisringes verlängert ist, sondern dadurch, dass die Basis (Abgangsstellen) der Fortsätze in den einzelnen Teilen verschieden weit zentralwärts auf der Innenfläche der Aussen- oder Scheiniris vorgeschoben wird (vgl. Zietzsehmann [11]). Am stärksten ist dies temporal der Fall, wo der spitzere Teil der eiförmigen Scheiniris liegt. Die funktionelle Iris stellt also einen etwa kreisförmigen Ausschnitt aus der Scheiniris̀ dar. Nach $\mathrm{Ab}$ zug dieses Ausschnittes bleibt von der Scheiniris, deren äussere Umrandung von den Spatia anguli iridis und den Irisfortsätzen gebildet wird, ein in den einzelnen Abschnitten verschieden breiter Ring übrig, von dessen Innenfläche Proe. cil. dem Linsenäquator zustreben. Dieser Teil der Scheiniris wird sich also (nach den oben erfolgten anatomisch-physiologischen Betrachtungen) nicht an der Verengerung oder Erweiterung der Pupille aktiv beteiligen können. Denn im andern Falle wïrde ja ein gegenseitiges Abhängigkeitsverhältnis zwischen Accommodation und Pupillenspiel bestehen. Wir können diesen fixierten Teil als "nichtfunktionellen" oder "ruhenden" Teil der Iris der "funktionellen" Iris gegenüberstellen. Der ",ruhende Teil" und die "funktionelle" Tris bilden zusammen die "Schein- oder Ausseniris".

(Bei rein physiologischer Auffassung ohne Rücksicht auf die anatomischen Verhältnisse, dje namentlich anch durch die Lage der grösseren Gefäss- und Nervenstämme gegeben sind, müsste man den Teil der Iris, von dessen Innenfläche Ciliarfortsätze abgehen, dem Accommodationsapparate, vielleicht der Grundplatte des Ciliarkörpers zurechnen.)

Betrachten wir jetzt ganz allein die funktionelle Iris, dann wissen wir aus dem vorhergehenden, dass ihre periphere Umgrenzung etwa Kreisform hat (Taf. IX, Fig. 1). Bei Miosis, also im ausgebreiteten Zustande, stellt die funktionelle Iris eine runde Scheibe dar (diese ist natürlich nicht flach, sondern stellt den Mantel eines ganz niedrigen, mit der Spitze nach aussen gerichteten Kegels dar). Wie die beigegebene Zeichnung (Taf. IX, Fig. 1) zeigt, ist diese Seheibe nicht direkt in der Mitte, sondern etwas ventral und temporal verschoben, von einem quergestellten, schmalen Spalt mit abgerundeten Enden durchbrochen. Bei Mydriasis sehen wir ein weites querovales Loch, das sich desto mehr der Kreisform nähert, je stärker die Dilatation ist. Bei Miosis hat also die Iris dieses rundliche Loch bis auf einen schmalen Spalt verschlossen, der auch hier etwas ventral und temporal von der Mitte der Öffnung bei Mydriasis gelegen ist. Die Länge des Spaltes bei Miosis in naso-temporaler Richtung, also der grössten Ausdehnung, ist nur wenig kleiner als der gleiche Durchmesser der Pupille bei Mydriasis. Hingegen differieren die Durchmesser der beiden Pupillen in dorsoventraler Richtung ausserordentlich (Taf. IX, Fig. 1). Zeichnet man sich die Konturen der funktionellen Irides desselben Tieres mit entgegengesetzter Pupillenstellung ineinander ein, so ist ohne weiteres ersichtlich, dass der Raum, der bei Miosis von der Iris vorhangartig verschlossen 
worden ist, dorsal und ventral bedeutend grösser ist als nasal und temporal an den Enden des Spaltes, und zwar ist die dorsal zu verdeckende Fläche wieder grösser als die ventrale und die temporale klemer als die nasale. Um einen solchen Verschluss zu ermöglichen, wird zunächst die Iris oben und unten an den beiden Langseiten des miotischen Pupillenspaltes, eine entsprechend grössere Quantität von Stoff zur Entfaltung des Vorhanges zur Verfügung haben müssen, als temporal und nasal. Dieser ausbreitungsfähige Stoff wird in der Hauptsache von dem Stroma iridis gebildet, und so finden wir dieses auch in überwiegender Quantität oben und unten angehäuft. Bei enger Pupille tritt dieses Verhalten auf den ersten Blick in der grossen Breitenausdehnung der Iris an den beiden Langseiten des Pupillenspaltes in Exscheinung; bei weiter Pupille sehen wir namentlich gut auf Radiürschnitten die grosse Menge. des Stroma dorsal und ventral in dicken Wülsten zwischen quer verlaufenden Furchen zusammengerafft. Die Radiärschnitte aus der Gegend der Enden des Pupillarspaltes zeigen dagegen die mydriatisehe Iris mehr keilformig mit glatten änsseren und inneren Flächenkouturen (Taf. X, Fig. 2-9). Noch bessel erkembar wird diese verschiedene Stoffverteilung des Stroma beim Vergleich yon Radiärschnitten aus den einzelnen Partien von Irides nit weiter und enger Pupille von ein und demselben Tiere. Wir sehen hierbei, dass sich die Radiärschnitte aus der Gegend des nasalen und temporalen Pupillarwinkels, bei enger und weiter Pupille temporal fast gar nicht, nasal nur wenig voneinander unterscheiden. Demgegenüber bieten sich die Untersehiede bei Miosis und Mydriasis in den pberen und unteren Teilen in ohne weiteres ersichtlicher. Weise dar und zwar sehen wir auch hier, dass dorsal die Dehnung bzw. Kontraktion ausgiebiger ist als ventral. In den dazwisehen liegenden Partien ist natürlich gemäss der kreisfömigen Umrandung der funktionellen Iris ein allmählicher Ubergang zu konstatieren. Es ist anzunehmen, dass ausser diesen stark in die Augen fallenden quantitativen Verseliedenheiten auch qualitative im Aufbau des bindegewebigen Stromas worhanden sein werden, die in den oberen und unteren Tellen eine leichtere und ansgiebigere Raffung bzw. Dehnung des Stromas ermöglichen. Ein histologischer, einwandfreier Beweis hierfür könnte nur auf Grund pingehender spezieller Cntersuchnogen erbracht werden. Sicher aber ist es, dass durch eine gewisse Anordnang und Lagerung der muskulösen Elemente der Iris namentlich des Dilatators das diesen als Medium dienende Stroma in den Seitenteilen der Iris, am meisten am temporalen Winkel, des Pupillenspaltes einen strafferen Chamakter erhält als oben und unten. (Das nähere über diesen Punkt findet sich in dem mikroskopischen Teile der Arbeit.) Hierin lisst sich sehon das Zusammenwirken des passiven Teiles der Iris, des bindegewebigen Strokas, mit dem aktiven oder mnskulösen durch Sphinkter and Dilatator gegebenen Teil erkennen.

Wir sind so von dem mehr passiven Teile des Irisblendapparates, der zunächst grundlegend fur das Zustandekommen dex querovalen bzw. spaltformigen Puplle ist, schon auf den aktiven muskulosen Teil der Iris geraten. Wir müssen annehmen, dass auch in seinem morphologischen Aufbau die Versehiedenheit der Aufgaben zum Ansdruck kommt, die an ihn in den einzelnen Abschnitten gestell werden. Wir wollen ons dis Aufgaben kurz 
vergegenwärtigen, die die beiden den Muskelapparat bildenden Muskeln, der Dilatator und Sphinkter, zu leisten haben. Dilatator und Sphinkter sind in ihrer Wirkungsweise Antagonisten. Für das passive Stroma sowohl wie fur den aktiven Muskelapparat wird es ein Ruhestadium geben, wo einmal das Stromagewebe keine Dehnung erfährt, anderseits Dilatator und Sphinkter sich in gegenseitiger Ruhelage befinden. Es ist wahrseheinlieh, dass ähnlich wie bei der Muskulatur am Skelett die beiden Antagonisten anch hier bei Ruhelage einen wenn auch nur geringen Zug aufeinander ausüben, also im Tonus liegen. Für beide Teile wird ungefähr bei derselben Pupillenweite die Ruhestellung erreicht sein. Die Kombination beider ergibt dann die Ruhestellung der Tris insgesamt. Analog wie Münch (9) beim Menschen habe ich auch beim Schaf festgestellt, dass die Ruhestellung der Iris bei einer geringgradigen Miosis, nicht bei mittlerer Pupillenstellung zu suchen ist. Um dies festzustellen, machte ich mit Schafaugen folgenden Versuch:

Ein frisches Auge eines geschlachteten Tieres, das stark erweiterte Pupillen zeigte, liess ich in einer feuchten Glaskammer vor Austrocknen geschützt einige Tage bis zur Lösung der Muskelstarre liegen. Beim Vergleich mit dem in Formalin fixierten zweiten Ange desselben Tieres und auch durch Bezeichnung der Pupillengrösse an der Cornea konnte ich feststellen, dass sicb die Pupille zu einer mittleren Pupillenstellung verengert hatte. Eröffnete ich nun den Bulbus von diesem Auge, legte die Iris durch Entfernung der Linse frei und liess das Präparat im Wasser schwimmen, so trat sogleich eine starke Miosis ein, etwa so wie 'Taf. IX, Fig. 1 zeigt. Eine mehrmalige Wiederholung dieses Versuches ergab immer das gleiche Resultat. Man kann daraus schliessen, dass die Iris des Schafes, wenn kein Muskeltonus vorhanden ist, bei starker Miosis ibre Ruhestellung hat. Dass diese Pupillenstellung bei unverletztem Auge nicht vollkommen zu stande kommt, erkläre ich mir daraus, dass die Linse durch ihre gewölbte Oberfläche, auf der die Iris geleitet, der vollständigen Verengerung ein Hemmnis entgegensetzt. Beim lebenden Tiere, wo noch Muskeltonus vorhanden, wird daher die Ruhestellung auch beim Schafe mehr bei Miosis zu suchen sein, wie es Münch für den Menschen annimmt. Versuche mit Augen von Hunden ergaben ein gleiches Resultat.

Bei Erweiterung der Pupille aus dieser Ruhestellung wird der aktive Dilatator einmal den Widerstand überwinden müssen, den der passive Teil der Iris, nämlich das Stroma bietet. Dieser ist gegeben durch den Aufbau seines aus Bindegewebe mit Gefässen und Nerven bestehenden Gerüstes und durch seine Anfüllung mit Blat und Lymphe. Daneben wird sich im Stroma noch ein geringer Gegenzug in mehr seitlich cirkulärer Richtung nach den temporalen und nasalen Enden des Pupillenspaltes geltend machen. Haben wir doch gesehen, dass beim Pupillenspiel die Iris in diesen Partien nur eine sehr geringe Formveränderung erleidet, was dort teils dureh die geringe Quantität von vorhandenem Stroma, teils durch die später zu besprechende Einlagerung von Dilatatorfasern erreicht ist. Die weiter temporal und nasal gelegene Masse des Irisstromas ist also stärker fixiert als die weiter nach der Mittellinie zu gelegene. Dadurch entsteht bei der starken Raffung der Iris in den mittleren Teilen des oberen und unteren Quadranten ein Gegenzug in Stroma nach den nasal und temporal ge- 
legenen fixierten Stellen hin. Einen zweiten Widerstand fur die Aktion des Dilatators bildet der ringförmig in sich geschlossene Sphinkter. Bei Mydria. sis werden seine erschlafften Muskelfasern gedehnt werden müssen. Da er infolge seiner Lage im Stroma gleichfalls in den Seitenteilen stärker fixiert ist, so wird sein Gegenzug die Richtung nach diesen temporal and nasal liegenden stärker fixierten Punkten haben. Die meiste Arbeit hat abel der Dilatator bel der Erweiterung der Papille in den mittleren Teilen des oberen und wnteren Quadranten zu verrichten, wie das aus der beigegebenen Zeichnung (Taf. IX, Fig. 1) hinlänglich orkennbar ist. Da nun bei ihm die Faserverlaufsrichtung mit seiner Wirknngsrichtung in der ganzen Circumferenz der Iris in der Hauptsache zusammenfäll, so wird der Dilatator auch in den Teilen des Irisringes am stärksten entwickelt sein, wo er die meiste Arbeit zu leisten hat, d. i. in den mittleren Parfien des oberen und unteren Quadranten. Nach der temporalen und nasalen Seite hin wird mit der Abnahme seiner zu leistenden Arbeit auch eine Verminderung in der Starke seiner Entwicklung zu konstatieren sein. Nun haben wir oben gesehen, dags bei Mydriasis in den Seitenteilen der Iris an den Fnden der querovalen bzw. spaltenförmigen Pupille die zentrifugale Bewegung der Tris (- Verschmälerung der Radiarbreite der funktionellen Iris) temporal fast $=0$, nasal nur gering ist. Danach milsste man annehmen, dass an den Enden des Pupillarspaltes nasal der Dilatator verhältwismässig gering, temporal aber so gut wie gar nicht entwickelt seir wird. Hier muss aber noch eine andere Erwägung Platz greifen. Die Randzone der Ir's ist infolge des dort in ihn eingelagerten Sphinkter's einem elastischen Ringe in Gestalt eines länglichen Querovales gleich zu achten. Bei Mydriasis werden die mittleren Teile del Langseiten" durch den dort starken Dilatator anseinander gezogen. Hierbei muss an den Enden des querovalen elastischen Ringes ein zentripetaler Zug zur Geltung kommen. Dieser wird bestrebt sein, die temporalen und nasalen Teile der Iris auch bei Mydriasis etwas zu nähern und den temporalen und nasalen Pupillendurchmesser zu verkleinern. Oben zeigte ich, dass am temporalen Pupilarende die Iris bei Mydriasis so gut wie gar keine Verschmälerung ihrer Radiärbreite erfährt. Hier wird also eine dilatierende Kraft wirken müssen, die zusammen mit dem Widerstand, den das Stroma seiner Debnung entgegensetzt, yener zentripetalen Kraft etwa die Wage hält. Am nasalen Ende der Pupille werden dilatierende Kräfte rorhanden sein müssen, die jeren zentripetalen Zng ubberwinden und noely eine geringe Zusammensehiebung des Stromas bewirken. Wir sehen also, dass auch die seitlichen (termporalen und nasalen) Purtien bei Mydriasis einer zentrifugal wirkenden Kraft bediurfen, wenn auch diese temporal nur in einer Art ron Hemmung in Erseheinung tritt. Wir werden weiter unten sehen, dass auch bei Miosis in ähnlioher Weise, aber in weit stärberem Masse diese Telle der Wris um die Enden des Pupillenspaltes auf zentripetalen $\mathrm{Zug}$ beansprucht werden, damit die Form des spaltfömigen Sehloches gewahrt wird.

Bei Verengernng der Pupille fallt die zu leistende Aufgabe dem Sphinktermuskel zu. En hat die querovale Pupille bei der Rukestellung der Iris, die wir bei schwacher Miosis angenommen, zu einem sehmalen Spalt zu verengern. Der Widerstand, den er dabei zu uberwinden hat, ist ein- 
mal gegeben durch das Stroma, das entfaltet und gedehnt werden muss, wobei ihm in geringem Grade der Druck des in die Gefässe einströmenden Blutes zu Hilfe kommen wird. Sodann hat er die erschlafften Muskelfasern des Dilatators zu dehnen; die vom Sphinkter zu leistende Arbeit ist demnach auch wieder in den mittleren Teilen des oberen und unteren Quadranten am stärksten, bedeutend kleiner nasal, und temporal fast gleich Null. Nun fällt aber beim Sphinkter einmal die Wirkungsrichtung mit seiner Faserverlaufsrichtung nicht zusammen, sodann bildet der Sphinkter ein in sich geschlossenes ringförmiges Muskelsystem. Infolgedessen wird die $z \mathfrak{u}$ leistende Arbeit, die in den einzelnen Abselmitten des Irisringes verschieden gross ist, auf den ganzen Muskelring gleichmässig verteilt werden können. Wir können daher von vornherein vermuten, dass auch beim Sphinkter trotz der versehiedengradigen Anforderungen, die auch an ihn in den einzelnen Abschnitten des Irisringes gestellt werden, doch keine grossen lokalen Verschiedenheiten vorherrschen werden.

Anderseits wird aber bei stärkerer Miosis auch durch die Kontraktionswirkung des Sphinkters auf die temporalen und nasalen Teile der Iris an den Enden des Pupillenspaltes eine starke zentripetale Kraft ausgeübt werden, wie bereits oben angedeutet. Wir seben also, dass ebenso wie bei Mydriasis auch bei Miosis, aber hier in noch weit höherem Grade diese seitlichen Partien auf zentripetalen Zug beansprucht werden. Da wir nach den obigen Ausführungen annehmen mussten, dass der Dilatator in diesen Partien nur gering entwickelt ist, so hätte das Stroma allein diesen Zug auszubalten. Ohne besondere auffällige Einrichtung oder Verstärkung wäre es jedoch hierzu nicht im stande. Wir müssen daher vermuten, dass in diesen Teilen des Irisringes gewisse Einrichtungen vorhanden sein werden, die der hier sowohl bei Mydriasis als auch noch stärker bei Miosis zentripetalen Kraft entgegenzuwirken und das Stroma zu unterstützen vermögen. Diese Einrichtungen müssten nasal, wo bei Miosis noch eine kleine Dehnung statt hat, in geringerem Grade entwickelt sein als am temporalen Ende des Pupillenspaltes, wo die Verbreiterung der Iris bei Miosis fast $=0$ ist. Wie dies durch eine gewisse Ausbildung des Dilatators in diesen Partien erreicht ist, werden wir im milkroskopischen Teile der Arbeit kennen lernen.

Im folgenden will ich nun genauer darstellen, was ich bei meinen mikroskopischen Untersuchungen in den einzelnen Partien des Irisringes feststellen konnte, namentlich am muskulösen Apparat, nämlich am Dilatator und Sphinkter. Ich tue dies, wie schon im technischen Teil erwähnt, an Hand von Schnittserien, die durch Schnittfürung in radiärer, tangentialer (transversaler) und in der dritten Raumrichtung parallel zur Oberfläche der Iris erhalten sind. Hierzu nahm ich zum Vergleich Irides sowohl mit weiter als auch mit enger Pupille.

Aus den oben gemachten Betrachtungen ergibt sich, dass wir auch hier bei der mikroskopischen Beschreibung die oberen und unteren Teile des Irisringes, die an den Langseiten des Pupillen- 
spaltes bzw. -ovales liegen, den temporalen und nasalen Teilen an den Enden des Pupillenspaltes gegenüberstellen werden.

Wie das die rundliche Umgrenzung der Iris ohne weiteres bedingt, werden natürlich die Hauptpartien, oben, nasal, unten, temporal allmählich ineinander ïbergehen und solche Übergänge sind natürlich auch in dem anatomischen Aufbau zu konstatieren.

\section{Oberer (dorsaler) Iristeil.}

I. Bei weiter Pupille.

Betrachten wir zunächst einen Radiärschnitt aus der Mitte des oberen Teiles des Irisringes bei weiter Pupille (Taf. X, Fig. 2). Nach meinen früheren Ausführungen beginnt die funktionelle Tris peripher am Scheitelpunkt des Winkels, der von der Innenfläche der Iris und den iuberragenden Teilen der Ciliarfortsätze gebildet wird. Von dem Scheitelpunkt des Winkels, den die Aussenfläche der Iris mit der Innenfläche der Cornea bildet, also von den Spatia anguli iridis liegt die Ciliargrenze der funktionellen Iris etwa $1 / 6-1 / 4 \mathrm{~mm}$ weiter zentralwärts entfernt. Diese minimale Breite hat also hier die sogenannte ruhende Iris, das ist die periphere Zone der Scheiniris nach Abzug der funktionellen Iris. Die Kontur der inneren, retinalen Fläche der Iris verläuft im Schnitte in ziemlich gerader Richtung bis zum Pupillarrande, dem das Traubenkom aufsitzt. Es finden sich nur ganz flache Ausbiegungen. Manchmal erscheint die Kontur in einer ganz flachen, kaum merkbaren S-form. Verfolgen wir die Kontur der Aussenfläche der Iris ron ihrer peripheren Grenze an, so sehen wir, dass diese einen grossen Bogen beschreibt, dessen peripherer Anstieg flacher ist als der pupillenseitige Abfall. Bei starker Mydriasis erscheint letzterer sogar häufig überhängend. Das Ende dieses Abfalles liegt etwas pupillenwärts uber der Mitte der Radiärbreite der funktionellen Iris, wobei ich das Traubenkorn als Aufsatz unberücksichtigt lasse. Ein ciliarer Randteil des Sphinkters, etwa $1 / 5-1 / 4$ der ganzen Sphinkterbreite, wird noch in diesen Bogen eingeschlossen. An den Abhängen des Bogens finden sich einige Einkerbungen, durch die sekundäre Bogen abgegrenzt werden. Die Höhe des Bogens beträgt etwa das Doppelte der Dicke der funktionellen Iris am Trisciliarfortsatzwinkel. In dem Teile der Iris, der von diesem Bogen umschlossen wird, gewahrt man immer die Querschnitte grösserer Gefäss- (Oirculus arteriosus und venosus iridis) und Nervenstämme. Hier finden sich anch an manchen Schnitten Bilder, die dadurch entstehen, dass die hier stark entwickelten Strukturfalten 
Der muskulöse Apparat der Iris des Schafes und seine Beziehungen usw. 419

und Dilatatorleisten in der Längsrichtung oder auch mehr schräg getroffen sind. Die genauere Darstellung dieser Bilder erfolgt weiter unten.

Die wulstartige Verdickung der Iris in der ciliaren Hälfte ist natürlich die Folge der durch den Dilatator veranlassten Raffung und Zusammenschiebung des Stromagewebes. Entsprechend der Lokalisation des Dilatators an der Innenfläche der Iris hat daher auch die Kontur dieser auf dem Radiärschnitte insgesamt eine geradlinige Richtung. Hierbei lasse ich die durch die aktive Kontraktion der Dilatatorelemente bedingte Raffung der inneren Retina- oder Pigmentschicht als eine in den Hintergrund tretende Wellung unberücksichtigt. Die Kontur der Aussenfläche der Iris vom pupillaren Ende des grossen Bogens an verläuft dann in mehreren flachen Bogen mit einer entsprechenden Anzahl von wenig tiefen Einkerbungen bis zum Pupillarrande bzw. Granulum iridis. Die Dicke des Irisquerschnittes in dieser Partie bleibt, abgesehen von den eben beschriebenen Unebenheiten, bis zum pupillaren Ende ungefähr gleich oder nimmt nur mässig ab. Sie beträgt etwa die Hälfte der Dicke der funktionellen Iris am Irisciliarfortsatzwinkel.

Der Sphinkter beginnt ungefähr an der Grenze zwischen dem zweiten und dritten Fünftel der Radiärbreite der funktionellen Iris vom Ciliarrand gerechnet. Ein peripherer Teil ron ihm liegt noch innerhalb der grossen Irisverdickung. Seine ersten kleinen vereinzelt liegenden Bündel, die hier dorsal im Irisring vom Schnitt vollkommen quer getroffen sind, liegen in unmittelbarer Nähe der pigmenttragenden Innenfläche. Die sich pupillenwärts anschliessenden Bündel entfernen sich allmählich von der Innenfläche bis in die Nähe des pupillaren Abfalles jenes grossen Bogens der Aussenkontur; dort werden die Bündel umfangreicher und liegen dichter nebeneinander. In scharfem Bogen oder Winkel begleiten sie den starken Abfall jenes grossen Bogens und treten dann wieder dicht an die Innenfäche heran. Weiter pupillar macht das Band, das von den Sphinkterbïndelquerschnitten gebildet wird, zum Teil die Bogen mit, die in dieser Gegend die Aussenkontur zeigt. Es entstehen so zwischen Sphinkter und Irisinnenfläche eine Anzahl flacher Nischen, wo mehr Stromagewebe den Sphinkter von den Pigmentschichten trennt. Weiter nach dem Pupillarrande zu sind die Sphinkterbündel mehr in kontinuierlich gleichem Abstande in der Nähe der Innenfläche gelagert. Der Sphinkter endigt dicht an dem Pupillarrande.

Der Dilatator iridis ist, wie wir wissen, an die retinalen Zell- 
schichten der Innenfiäche gebunden. Bezüglich seines feineren Aulbaues beim Schafe verweise ich auf die Arbeit von Klinge (8). Seine Kontraktion bei Mydriasis bewirkt es, dass wir auf unsern Radiärschnitten die Kontur der Innenfläche in geradliniger Richtung verlaufen sahen. In dem dorsalen Viertel des Irisringes, den wir jetzt betrachten, erfährt der Dilatator durch ausgiebige Bildung von Struktur-falten und Dilatatorleisten entsprechende Verstärkung. Eine genauere Schilderung von diesen Bildungen gebe ich weiter unten an der Hand von Tangentialschnittserien, wobei ich auch auf die Bilder eingehen werde, die sich auf Radiärschnitten zeigen. Hier mögen über diese nur folgende Andeutungen Platz finden. In manchen Radiärschnitten verlaufen die beiden. inneren Irisschichten glatt in einfacher Anordnung. In vielen Schnitten sehen wir aber durch Einbuchtung und Einstrahlung entstandene Unregelmässigkeiten der hinteren Kontur auftreten, auf die auch schon K.linge(8) hingewiesen hat. Es sind dann von dem Schnitte Strukturfalten teils in ibrer Verlaufsrichtung, teils etwas schräg zo ihr in grösserer oder kleinerer Ausdehnung getroffen. Hier lassen sich fast immer an diesen ins Stroma einspringenden Buchten und Strahlen die Zellen beider Retinaschichten nachweisen. Neben diesen Bündeln sehen wir aber auch pigmentierte Streifen obne direkten Zusammenhang mit der Innenflächenkontur im Stroma ungefähr in radiärer Richtung mehr oder weniger parallel zur Innenfächenkontur verlaufen. Es sind das in der Regel längsgetroffene Dilatatorleisten. Doch kann auch ein solches Bild entstehen, wenn eine Strukturfalte scharf tangential nur in der äusseren Dilatatorlamelle getroffen ist. An diesen Streifen sind nur die Zellen dieser äusseren Retinalamelle nachzuweisen. Die Streifen finden sich namentlich in der ciliaren Hälfte des sphinliterfreien Iristeiles. Längs getroffene kleinere Strukturfalten sehen wir aber auch hinter dem Sphinkter soweit pupillenwärts, als sich von den Begen der Sphinkterbündelreihe umgrenzt jene Stromanischen vorfinden. Diese Nischen sehen wir auch manchmal in mehr schräg radiärer Richtung durchzogen werden won pigmentierten Streifen, die aus längsgetroffenen Zellen der Dilatatorschicht bestehen. Auch finden sich hier im Stroma hinter dem Sphinkter kleine schwache pigmentierte Muskelzüge, die quer oder schräg getroffen sind. Auf die Deutung dieser Bildung komme ich unten noch genauer zu sprechen.

An der Hand von Tangentialschnittserien und der Heranziehung der Befunde in den Radiär- und Flachschnitten will ich nun eine genauere Schilderung des Dilatators und seiner Verstärkung im mitt- 
leren Teile des oberen Quadranten geben. Der Übergang der Ciliarfortsätze in die Iris findet unter Bildung eines Winkels statt, wenn man einen Radiärschnitt betrachtet (Taf. X, Fig. 2), oder unter Zustandekommen einer cirkulären Rinne, wenn man den ganzen Irisring berücksichtigt. Die tiefste Partie dieser Rinne ist zugleich der Grund der ringförmigen peripheren Nische der hinteren Augenkammer. Nun ist dieser Grind keineswegs glatt und mit der flachen Ausbreitung der beiden Retinalamellen ausgelegt. Er ist vielmehr ganz unregelmässig ausgebuchtet und ausgezackt. Hierbei trennen sich häufig die beiden Retinalamellen voneinander. Manchmal entsteht ein richtiges Maschenwerk, an dessen Zustandekommen sich auch Bindegewebe mit Gefässen beteiligen. Diese Unregelmässigkeiten erstrecken sich natürlich auch auf den irisseitigen Abhang dieses Grabens. Hier macht sich jedoch in den Unebenheiten eine mehr radiäre Anordnung geltend, und dies ist der Beginn der radiären Strukturfalten und Dilatatorleisten; denn an dieser Stelle beginnt auch an der äusseren Lamelle sich die Bruchsche Membran auszubilden. Verfolgt man eine Serie von Tangentialschnitten vom Ciliarkörper her pupillenwärts, so sind da, wo die Trennung von Ciliarkörper und Iris vollständig geworden ist, schon ziemlich starke Unebenheiten in der hinteren Profillinie zu konstatieren. Es finden sich unregelmässige, wellenförmige Aus- und Einbuchtungen. Die Einfaltungen der Innenfläche können teils flach sein, teils mehr rinnen- und spaltenartig in das Stroma einspringen. Einzelne dieser Spalten können schon hier bis $1 / 8(1 / 6)$ der Irisdicke in das Stroma hineinragen. Neben diesen radiären Strukturfalten, die von beiden Lamellen ausgekleidet sind, macht sich auch schon eine besondere Faltenbildung an der Dilatatorlamelle allein geltend. Es sind hier alle Übergänge vorhanden, von flachen Vorwölbungen, knospenartigen Vorsprüngen bis zu kurzen Zapfen. Man kann sie an allen Stellen der inneren Irisfläche finden, sowohl an der Spitze und an den Seiten der ins Stroma vorspringenden Radiärfalten, wie auch in den flachen Tälern und Wölbungen der unregelmässigen Innenfläche. Die Dilatatorleisten sind also nicht in irgend einer Weise an die radiären Strukturfalten gebunden. Das Studium der Radiärschnitte bestätigt das frühe Auftreten der radiären Strukturfalten und Dilatatorleisten in der Nähe des Ciliarkörpers. Man sieht nicht selten in Radiärschnitten in der Nähe des Irisciliarfortsatzwinkels Strukturfalten und Dilatatorleisten auf kleinere oder grössere Strecken getroffen. Sie treten dann als pigmentierte Streifen auf, die je nach der Tiefe der Falten mehr oder weniger 
weit von der Innenfläche entfernt im Stroma ciliarwärts verlaufen. Dieses Bild kann den Anschein erwecken, als ob der Dilatator in den Ciliarkörper ausstrahle. Wenn man aber die Serie von Schnitten weiter verfolgt, stellt sich diese Annahme als Irrtum heraus. Diese Streifen sind niemals ciliarwärts weiter über den Irisciliarfortsatzwinkel hinans zu verfolgen; vielmehr sieht man nicht selten, wie sie nach dem Ciliarkörper hin bogenförmig und mehr oder weniger parallel zur Profillimie des Irisciliarkörpergrabens umbiegen [wie das auch Klinge (8) beschreibt] und sich in der Retinaschicht des Ciliarkörpers oder in dem dort sich erentuell vorfindenden oben erwähnten Maschen. werk verlieren.

Bei der Durchsicht der entsprechenden Serie von Flachschnitten wird die Auffassung, die man durch das Studium der Serien in den beiden andern Richtungen gewonnen hat, in jeder Weise gefestigt. Wie verhalten sich nun die radiären Struktur- und Dilatatorfalten in ihrem weiteren Verlaufe nach dem Sphinkter zu? Verfolgen wir wieder die Reihe von Tangentialschnitten, so sieht man, dass Strukturfalten und Dilatatorleisten ziemlich rasch an Ausbildung und Zahl zunehmen. Will man die Gegend der stärksten Ausbildung dieser Dilatatorverstärkung ungefähr angeben, so ist sie etwa in der Mitte zwischen Ciliarfortsatziriswinkel und ciliarem Sphinkterrande gelegen. Von dort ab nimmt die Stärke der Ausbildung namentlich der Dilatatorleisten nach dem Sphinkter zu schneller ab als ciliarwärts, wo die Falten bis nahe zu dem Winkel hin ziemlich tief in das Stroma einschneiden. Wie bereits oben erwähnt finden wir an dieser Stelle der stärksten Ausbildung der Dilatatorleisten und Strukturfalten an den Radiärschnitten den Querschnitt staxk verbreitert and das Stroma wulstartig vorgewölbt. Im mittleren Teile dieser Wölbung sahen wir auch die Querschnitte der grössten Gefäss- und Nervenstämme. Aber anch nach Abzug des Einflusses, den diese Gefässe hier auf die Stromadicke ausïben können, ist die Annahme voll gerechtfertigt, dass die Anhäufung des Stromagewebes in dieser Partie sich erklären lässt durch eine besonders ausgiebige Kontraktion des Dilatators in dieser Gegend der stärksten Ausbildung der Struktur- und Dilatatorfalten. Wie stellen sich nun die Verstärkungsfalten des Dilatators in dieser Partie ihrer stärktsen Entwicklung dar? Bei den radiären Strukturfalten, die von den beiden retinalen Lamellen ausgekleidet sind, finden wir auf dem Tangentialschnitt betrachtet alle Utbergänge von flachen Ausbuchtungen über engere, nicht immer spaltförmige Einschnitte bis zu engen Zapfen, bei denen die freien 
Rücken der Zellen der inneren Lamelle ohne Zwischenraum dicht aneinander grenzen. Manchmal weichen aber auch in diesen Zapfen die beiden Schichtenpaare mehr oder weniger weit auseinander, so dass ein Hohlraum entsteht. Sie können bis $1 / 3$ der Irisdicke in das Stroma eingefaltet sein. Diese Einfaltungen sind immer nur einfach, sie zeigen niemals sekundäre Falten. Auf den Radiärschnitten präsentieren sich die Strukturfalten, je nachdem sie vom Schnitte mehr oder weniger. schräg zu ihrer Verlaufsrichtung getroffen sind, als radiäre breitere und schmalere Streifen, die im Stroma losgetrennt verlaufen, oder als in Streifen auslaufende Buchten, die in vorwiegend radiärer Richtung ins Stroma einspringen. Diese Streifen und Buchten sind aussen nach dem Stroma hin von der Bruchschen Membran begrenzt, dann folgt die äussere retinale Zellschicht mit ovalen oder kurz stäbchenförmigen Kernen und dann die mit mehr rundlichen Kernen ausgestattete Zellreihe der inneren Retinaschicht [vgl, auch Klinge(8)]. Die Flachschnitte bieten ein entsprechendes Bild. Die Strukturfalten exscheinen hier als radiär verlaufende rostartig angeordnete Zellstreifen, die jederseits aus den beiden Retinaschichten bestehen. Häufig trennen streifenartige freie Zwischenräume die doppelte Lage der beiden hinteren Irisschichten. Man kann ein schwaches Konvergieren dieser Streifen pupillenwärts nach der Mitte zu konstatieren. Auch hier auf den Flachschnitten sieht man die kleineren radiären Strukturfalten hinter dem Sphinkter.

Die Dilatatorleisten, die durch Einfaltung der Dilatatorlamelle allein gebildet werden, sind hier auch in Zahl und Stärke gut entwickelt. Auf dem Tangentialschnitte sieht man nebeneinander alle Übergänge von knospenartigen Vorwölbungen über kleinere Vorsprünge bis zu langen Zapfen, die ebenfalls bis $1 / 3$ der Irisdicke in das Stroma hineinragen können (vgl. Taf. X, Fig. 10). Sie sind fast nie gleichmässig dick mit parallelen Rändern, ihre Kontur ist meist unregelmässig ein- und ausgebuchtet. Häufig finden sich an den Zapfen noch kleine knospenartige sekundäre Auswüchse und etwas längere Fortsätze, also sekundäre Leisten. So kommt auf dem Tangentialschnitt eine Zeichnung zu stande, die mit Korallenbäumchen eine Ähnlichkeit hat. Die Leisten können in das Stroma hineinragen, ohne dass an ihrer Basis die Kontur des inneren Pigmentepithels beeinflusst wird. Häufig wird jedoch an den Stellen, wo die Leisten abgehen, auch die innere Retinalamelle mehr oder weniger stark eingebuchtet oder im Schnitt trichterförmig eingezogen. Es kommt so zur Bildung kleinerer Strukturfalten. Die Zellen der inneren Retina- 
schicht koonnen aber auch nur an der Basis der Dilatatorleisten mehr oder weniger stark angehäuft erscheinen. Manchmal gehen diese Leisten von dem Gipfel der Strukturfalten $a b$, so dass sie diese fortsetzen. Ebenso häufig sind aber auch die Seitenränder der Strukturfalten mit Buckeln und Knospen und längeren Auswüchsen der Dilam tatorlamelle besetzt. Es kommt dann ein Bild zu stande, wie es Grynfeltt (5) sehr zutreffend schildert.

Es finden sich in den Leisten häufig Hohlräume. Sie können klein und punktförmig sein, doch auch grösser, mitunter so gross, dass sie den zapfenförmigen Querschnitt einer Dilatatorleiste zu einem geschlossenen Ringe umgestalten können. Sie krommen überall in den Leisten vor, ohne Rücksicht auf die Grösse dieser, sowohl in den weit einspringenden Leisten, als auch schon in den kleinsten knospenartigen Buckeln. Man kann sie auch an allen Stellen der Leiste von der Basis bis zur Spitze hin antreffen, mancbmal mehrere hinter. einander. Der Leistenquerschnitt bekommt dann das Aussehen einer kurzen Kette von aneinander gereihten unregelmässigen Ringen. Die Hohlräume sind auch ebenso häufig an Stellen, die glatt und frei von Einfaltungen sind, zwischen den beiden Retinazellschichten zu finden. Die kleineren Hohlränme sind in der Leiste nur eine kürzere Strecke radiär zu verfolgen, schon etwas weiter die grösseren Hohlräume. Den ganzen Verlauf der Leiste habe ich sie nie durchsetzen sehen. Ich hebe dieses Verhalten bei Mydriasis Klinge gegenïber hervor, der diese Röhrenbildung als̀ eine Wirkung der Miosis betrachtet, während bei mydriatischer Stellung die Leisten nur als solide Gebilde geschildert werden.

Als Charakteristikum dieser Hohlräume lässt sich nur sagen, dass sie überall dort auftreten können, wo die äussere Pigmentzellschicht vorhanden ist. Manche Iris zeigt diese Hohlräume stark, manche schwächer ansgebildet. In verschiedenen Teilen ein und derselben Iris sind sie aber immer in ungefähr gleichem Grade ausgebildet. Ich habe sie bei Irides mit weiter Pupille weit stärker entwickelt gesehen als bei Miosis. Ich muss daher der Ansicht Klinges entgegentreten, der in diesen Hohlräumen in den Leisten, also in dieser Röhrenbildung eine Wirkung der Dehnung erblickt. Die Mutmassung scheint meiner Ansicht nach eine grössere Berechtignng zu haben, dass diese Hohlräume durch eine sekretorische Tätigkeit in den Zellen der äusseren Retinaschicht entstanden sind, vielleicht zum $Z$ wecke der Bildung des Kammerwassers.

In den Radiär- und Flachschnitten treten die Leisten als ähn. 
liche radiäre Streifen auf wie die radiären Strukturfalten, nur dass natürlich in der Mitte des Streifens die Zellen der inneren Pigmentschicht fehlen. Ebenso klar ist es, dass in ihren Randteilen getroffene Strukturfalten auf dem Radiärschnitte eine Leiste vortäuschen können. Einzelne Leisten und Strukturfalten kann man von ihrem Beginn in der Nähe des Ciliarfortsatziriswinkels bis zu ihrem Aufhören in der Nähe des peripheren Sphinkterrandes verfolgen. Daneben gibt es aber solche, die man nicht durchgehend oder auch nur kurze Strecken weit verfolgen kann. In der Regel sind die am tiefsten einspringenden Falten auch in der radiären Ausbildung am grössten. Verfolgt man wieder die Serie von Tangentialschnitten pupillenwärts, so sieht man, je näher man dem Sphinkterrande kommt, wie die Dilatatorfalten immer kleiner werden. Die kleinen Knospen und Fortsätze an den Leisten und Strukturfalten verschwinden; die Konturen werden glatter, die höheren Dilatatorleisten werden niedriger; sie verschwinden entweder schliesslich ganz oder wandeln sich dadurch, dass sich die innere Lamelle nun miteinbuchtet, in Strukturfalten um. Schliesslich beobachten wir nur noch Strukturfalten. Aber auch die hier vorhandenen Strukturfalten schneiden nicht mehr so tief in das Stroma ein. Die engen Spalten erweitern sich, die Buchten verflachen sich immer mehr, und in der Nähe des ciliaren Sphinkterrandes sieht man nur noch dicke kolbige Fortsätze der beiden Pigmentlamellen in das Stroma hineinragen. Die Leisten strahlen also hier nicht, wie Klinge behauptet, in den Sphinkterrand aus. Auch ist die hintere Kontur der Iris im ganzen betrachtet nur noch schwach gewellt und eingekerbt. Manche der pupillaren Ausläufer der Strukturfalten springen auch häufig nicht mehr senkrecht in das Stroma ein, sondern mehr oder weniger schräg nach der cirkulären. Richtung (Sphinkterfaserverlaufsrichtung) abgebogen. Es werden so durch die mehr oder weniger schräg einspringenden Strukturfalten rundliche Vorwölbungen an der hinteren Iriskontur unvollständig abgeschnürt. An den hier vorhandenen Strukturfalten, die .auf dem Tangentialschnitt meist als kurze kolbige Vorsprünge erscheinen, sieht man nun kleine pigmentierte Streifen in vorwiegend cirkulärer Richtung abstrahlen. In den nächsten Schnitten verlängern sich diese Fasern, treten mit den benachbarten in Verbindung, legen sich zu ganzen cirkulären. Faserzügen aneinander und rücken immer mehr von der hinteren Irisgrenze fort in das Stroma hinein. So beginnt der Sphinkter. Er legt sich also hier eng an die beiden hinteren Trisschichten an und entwickelt sich aus der äusseren Retinaschicht. 
Dieses Verhalten kann man auch auf dem Radiärschnitte bestätigt finden. Denn auch hier sieht man den ciliaren Rand des Sphinkters ganz dicht an die inmeren Irisschichten herantreten. Die Zellen dieses Anfangsteiles des Sphinkters sind pigmentiert wie die Zellen der Dilatatormembran, nur mit dem Unterschiede, dass die Pigmentkörnchen hier in dem spindelförmigen Zelleibe gleichmässiger verteilt zu sein scheinen. Auf dem Flachschnitte kann man ausserdem noch beobachten, wie dünne Fasern des Dilatators in kurzen Bogen in die cirkulär verlaufenden Sphinkterfasern einbiegen. Es sind das jene Strahlen, die wir auf dem Tangentialschnitt in schräger Richtung von den Strukturfalten am ciliaren Sphinkterrande ausgehen sahen.

Die Strukturfalten selbst sotzen sich auch noch hinter dem Sphinkter nach dem Pupillarrande zu immer niedriger werdend fort. Hier sehen wir aber auf Tangentialschnitten und Flachschnitten, wie überall von der Dilatatorlamelle Verbindungsfasern aus pigmentierten Muskelzellen zusammengesetzt in scharfen Bogen in den Sphinkter einstrahlen. Abgespaltene Sphinkterfasern kommen diesen Dilatatorfasern entgegen, so dass Sphinkter und Dilatator überall vom ciliaren Sphinkterrande bis zur pupillaren Endigung des Dilatators durch ein feines Maschenwerk von. Muskelfaserziugen in lockerem Zusammenhang stehen. Der Verlauf dieser muskulösen Verbindungsfasern in radiärer (ciliopupillarer) Richtung ist nur kurz. Sie erstrecken sich am weitesten in cirkulärer Richtung. Auf Radiärschnitten werden wir sie also in mehr querer Richtung getroffen sehen. Wir fanden sie als quer oder etwas schräg getroffene kleine pigmentierte Zellhäufchen im Stroma zwischen Sphinkter und Trispigment liegen. Daneben sieht man aber noch in den Radiärschnitten ebenfalls hinter dem. Sphinkter əine grössere Anzahl von längeren und kürzeren Faserzügen, die in schräg radiärer Richtung, also nicht scharf cirkulär abbiegend, von der Dilatatormembran nach dem Sphinkter hinziehen. Meist durchsetzen sie in schräger Richtung die Stromanischen hinter dem. Sphinkter, die, wie wir oben geschildert, dadurch entstanden sind, dass der Sphinkter namentlich in seiner ciliaren Hälfte bei Mydriasis in Bogen verläuft. Und wir gehen wohl nicht fehl, wenn wir annehmen, dass beim Zustandekommen dieser faltigen Anordnung des Sphinkters diese Radiärfasern eine aktive Rolle spielen. In der am weitesten ciliarwärts gelegenen und auch grössten Sphinkternische tritt manchmal ein besonders stark entwickelter radiärer Muskelzug in Erscheinung.

Die mittlere Partie des oberen und, wie wir später sehen werden, auch des unteren Trissektors zeichnet sich einmal durch starke Ent- 
wicklung des Dilatators aus entsprechend der ausgiebigen Beweglichkeit. Sodann finden wir aber auch hier am Dilatator und an seiner Verbindung mit dem Sphinkter eine.derartige Anordnung, dass der Ausbreitung der Iris bei Miosis im Muskelapparat ein möglichst geringer Widerstand entgegengesetzt wird. Denn die Verstärkung des Dilatators ist in diesen Partien durch lockere Faltenbildung (Strukturfalten und Dilatatorleisten) erfolgt, so dass eine Entfaltung dieses Teiles der Iris leicht von statten gehen kann. Die Verbindung des Dilatators mit dem Sphinkter durch jene bogigen Abstrahlungen und durch die radiären Verbindungsfasern, die aber nur eine geringe Ausdehnung in radiärer Richtung zeigen, hat auch einen mehr losen Charakter. Auf der andern Seite kann bei Erweiterung der Pupille hier eine ausgiebige Raffung der Tris in ihren einzelnen Teilen leicht erfolgen. Die stärkste Raffung erfolgt in der ciliaren Hälfte. Doch auch der Teil der Iris, der vom Sphinkter eingenommen wird, erfährt noch eine teilweise, wenn auch nicht so starke Zusammenschiebung. Es kommt durch diese jener bogige, wellenförmige Verlauf des Sphinkterbandes zu stande, den wir auf den Radiärschnitten fanden.

Da im temporalen und nasalen Quadranten des Irisringes andere Verhältnisse vorliegen, so muss, wie bereits vorn angedeutet, ein Übergang zu diesen vorhanden sein, der hier noch zu schildern ist. Die ungefähre Grenze, bis zu der echte Einfaltungen der Dilatatorlamelle (Dilatatorleisten) und stark entwickelte Strukturfalten zu finden sind, ist aus beigegebener Zeichnung (Taf. IX, Fig. 1) zu ersehen. Vom ganzen Irisringe nimmt dieser Teil etwas weniger als ein Viertel ein. Es findet sich eine Art Übergangszone. Die Leisten sind hier klein und spärlich oder fehlen ganz. Die Strukturfalten sind ebenfalls in Zahl und Stärke reduziert. Zugleich finden sich schon Andeutungen jener Form der Dilatatorverstärkung, die wir in den beiden Seitenteilen der Tris vorfinden. Am stärksten ist das Überspielen dieser Verhältnisse in die Grenzzone auf der temporalen Seite, da dort die andere Form der Dilatatorverstärkung stärker entwickelt ist als nasal (vgl. unten).

\section{Bei enger Pupille.}

Betrachten wir zunächst wieder die Radiärschnitte (Taf. $\mathrm{X}$, Fig. 3), so fällt uns sogleich die starke Verlängerung und Versehmälerung der Iris auf. Die Radiärbreite der funktionellen Iris ist ungefähr $21_{4}$ mal grösser als bei Mydriasis. Die Streckung der Iris ist namentlich in ihrer ciliaren Hälfte erfolgt. Die wulstartige An- 
häufung des Stromas in diesem Teile ist fast ganz rersehwunden. Nur an der Stelle, wo die Querschnitte der grösseren Blutgefässe und Nerven liegen, zeigt manchmal das Stroma noch eine flache Verdickung. Die Konturen der Aussen- und Innenfläche verlaufen gestreckt, nur ganz wenig geschweift. Die Kontur der Aussenfläche ist glatt, ohne Wölbung und Einkerbung. Bis zum ciliaren Sphinkterrande hin hat oft die Iris die gleiche Breite, nämlich die am Irisciliarkörperwinkel, die konstant geblieben ist. Es kann sich jedoch auch in der Gegend, wo die Querschnitte der grösseren Gefässe und Nervenstämme liegen, eine grössere Breitenausdehnung im Radiärschnitt erscheinen, wie bereits oben angedeutet. Im Bereich des Sphinkters wird die Tris allmählich dünner, bis etwa zur Hälfte jener Anfangsbreite. Die Kontur der Aussenfläche verläuft in diesem vom Sphinkter eingenommenen Teile auch nur in einer ganz flachen Wellenlinie; denn auch der Sphinkter hat eine Streckung erfahren, die namentlich in den mittleren Teilen deutlich ist. Dort ist jener stark wellenförmige Verlauf des Sphinkterbandes vollkommen verschwunden, und dieses liegt in grösserer Nähe der Innenfläche.

Die Strukturfalten an der Innenseite sind fast vollständig verstrichen. In der ciliaren Hälfte des sphinkterfreien Teiles treffen wir im Radiärschnitt hin und wieder noch eine schwache Andeutung einer längsgetroffenen Strukturfalte von geringe: Tiefe an. In dieser Gegend finden wir auch in manchen Schnitten längsgetroffene Dilatatorleisten als schwache Pigmentstreifen, die aber immer in unmittelbarer Nähe der Tnnenfläche verlaufen. In nächster Nähe des ciliaren Sphinkterrandes sehen wir in manchen Schnitten, wie ein Pigmentstreifen von der sonst gerade verlaufenden hinteren Kontur in radiärer Richtung nach dem Randbündel des Sphinkters spitzwinklig einspringt. Wie dieses Bild zu deuten und zu stande gekommen ist, werdeh wir unten bei Besprechung der Tangential- und Flachschnitte arkennen.

Die radiären Verbindungsfasern zwischen Sphinkter und Dilatator, die wir bei Mydriasis namentlich in den Stromanischen hinter dem bogigen Sphinkter liegen sahen, sind hier gleichfalls in gespanntem Zustande in dem jetzt schmalen, gleichmässig breiten Stromastreifen zwischen Irisinnenfläche und Sphinkterband zu erkennen.

Bei der Durchsicht der Serien von Tangentialschnitten erkennen wir, wie durch die starke Ausdehnung unseres Teiles der Tris sowohl in radiärer wie in seitlicher Richtung fast alle ausgeprägten Strukturfalten ausgeglichen sind. In der Nähe des Irisciliarfortsatzwinkels 
zeigen sich noch kleine Einschnitte und Einkerbungen ins Stroma. Diese gehen weiter pupillenwärts immer mehr und mehr in flachere Unebenheiten der Innenflächenkontur über. In der Nähe des Sphinkters streckt sich die Innenfläche immer stärker, so dass sie stellenweise ganz glatt und geradlinig verläuft. In nächster Nähe des Sphinkterrandes machen sich spitzwinklig einstrahlende schmale Einschnitte bemerklich. Nach dem Sphinkterrande zu werden sie immer tiefer und springen immer spitzwinkliger-und immer enger an die hintere Iriskontur angeschmiegt ins Stroma ein. Diese spitzwinkligen Einsprünge sind die Umformung jener rundlichen Abschnürungen bei Mydriasis, die wir damals in der Nähe des Sphinkterrandes gesehen haben. Sie waren durch die scharf cirkulär abbiegenden Verbindungsfasern des Dilatators zum Sphinkter hin hervorgerufen. Durch die Kontraktion der Sphinkterfasern und die dadurch erfolgte Anspannung der Verbindungsfasern sowie durch die Dehnung des ganzen Stromas sind diese bogenförmigen Abschnürungen zu flachen, gleichsam gebügelten Falten und Einschnitten umgewandelt. Mit der Spitze sind diese Einschnitte auf dem Tangentialschnitt nach den Seiten, also nasal und temporal gerichtet. In der Mitte liegt eine ganz schmale indifferente Zone, an deren Rande die temporal und nasal gerichteten Querschnitte mit ihrer Basis gegeneinander zeigen. Auf den Radiärschnitten haben wir diese Falten als Pigmentstreifen gesehen, die in der Nähe des ciliaren Sphinkterrandes spitzwinklig nach den Randbündeln des Sphinkters einsprangen. Hat man nur allein einzelne Radiärschnitte vor sich, so kann man leicht diese Gebilde als Speichenfasern auffassen, und es ist leicht möglich, dass Klinge durch sie zu der Annahme verleitet worden ist, die Dilatatorleisten gingen pupillar in Speichenfasern über.

Die nach den Seiten divergente Richtung der eben erwähnten Einschnitte bzw. Einfaltungen der Innenfläche werden verständlich, wenn man sich die Wirkung des Sphinkters vergegenwärtigt. Dieser hat seine fixen Punkte temporal und nasal an den Enden des Pupillenspaltes. Bei seiner Kontraktion wird sich in seiner Masse eine Bewegung und ein Zug nach diesen Punkten hin bemerkbar machen. Die bogigen Verbindungsfasern zwischen ihm und dem Dilatator, die in gewissem Sinne eine Insertion der Sphinkterfasern an der Dilatatorlamelle darstellen, werden gleichfalls in diesen Richtungen angespannt werden und so auch eine entsprechende Einziehung an der Innenfläche hervorrufen. Weiter pupillenwärts hinter dem Sphinkter sieht man, wie jene auch hier allenthalben rom Dilatator in kurzem 
Bogen in den Sphinkter einbiegenden pigmentierten Muskelfasern angespannt und ganz in die Faserverlaufsrichtung des Sphinkters hineingezogen sind. Auch die Flachschnitte zeigen ein entsprechendes Bild (vgl, unten).'

Welche Umwandlung haben nun die Dilatatorleisten bei Miosis erfahren? Die kleineren Buckel und Vorsprünge haben sich durch die allgemeine Dehnung der Iris nach allen Seiten ganz ausgeglichen. Die mittleren Falten haben nicht einen vollständigen Ausgleich orfahren; man sieht manchmal die Dilatatorlamellen in flacheren oder gewölbteren Bogen in das Stroma vorspringen. Die gedehnte und gespannte innere Lamelle überbrückt dann diesen Graben, während die Zellen der äusseren Retinalamelle ihn auslegen. Die tieferen Leisten sind im Querschnitt dïnner geworden und zeigen stellenweise auch solche Hohlräume, die durch Zugwirkung entgegengesetzter Richtung entstanden sind. Doch sind diese Hohlräume nicht ständig zu finden. Vielmehr liegen ebenso häufig die Pigmentzellen ohne Zwischenraum so zusammen wie bei Mydriasis. Ich kann daher der Behauptung Klinges, dass sich die Dilatatorfalten bei Miosis in Röhren umwandeln, in bezug auf das Schaf keineswegs beistimmen. Ich fand, wie schon oben erwähnt, solche Hohlräume auch in der Iris des Schafes bei Mydriasis in etwa gleicher Ausbildung. Sind solche Hohlräume in der. Iris präformiert, so werden sie natürlich anch bei Miosis in Erscheinung treten. Wir finden daher in diesem Stadium die Hohlräume auch meist mehr oder weniger seitlich verzogen und abgeplattet, nicht rund als Röhrenquerschnitt; denn bei der Ausbreitung ist das Stroma mit dem eingelagerten Muskelapparat Zug- und Druckkräften nach den verschiedenen Richtungen hin unterworfen. Diese werden natürlich auch bei der Umgestaltung der grösseren Dilatatorleisten von entscheidendem Einfluss sein. Wir wollen uns daher kurz vergegenwärtigen, in welchen Hauptrichtungen diese Kräfte in den einzelnen Teilen unseres Irissektors wirken. Zunächst wirkt eine Zugkraft in radiärer Richtung; sie wird veranlassen, dass die einzelnen Zellen der Dilatatorlamelle in die Länge gezogen und dadurch ihr Querschnitt verkleinert wird. Daraus ergibt sich wieder ein Dünner- und Niedrigerwerden der Leisten auf dem Querschnitt. Zu gleicher Zeit wird dio Dicke der Iris verringert. Die Leisten werden also gegen die straffere Innenfläche angedrückt. Weiterhin wird bei Verengerung der Pupille durch die ausgiebige Ausbreitung und Bewegung der Iris in den mittleren Teilen gegenïber den mehr fixeren seitlichen Partien sich im Stroma ein Zng nach der Mittel- 
linie zu bemerkbar machen. Material zur Entfaltung des Irisvorhanges wird nach den Stellen des grössten Ausschlages gleichsam von den Seitenteilen herbeigeholt. Diese nach der Mittellinie gerichteten Zugkräfte werden im Verein mit der vorigen Wirkung der Verflachung (Abplattung) der Iris in den mehr temporal und nasal gelegenen Teilen des Irisstromas die dort befindlichen Leisten im ganzen nach der Mitte zu umlegen bzw. abbiegen. In den mittleren Partien des Irissektors werden sich die gegen die Mittellinie gerichteten Zugkräfte mehr oder weniger gegenseitig aufheben. Es werden daher die senkrecht zur Irisfläche wirkenden Kräfte, die die Verflachung und das Dünnerwerden der Iris verursachten, fast nur noch allein zur Geltung kommen. Die hier befindlichen Leisten werden also gegen die straffe Innenfläche gestaucht, abgeknickt oder zusammengefaltet. Und so beobachten wir auch im Tangentialschnitt, wie die Gipfel der Falten in den mehr temporal und nasal gelegenen Partien nach der Mittellinie des fraglichen Sektors zu weisen und die Falten selbst einen spitzen Winkel mit der hinteren Iriskontur bilden, der gleichfalls nach der Mittellinie des Sektors zu offen ist. In den mittleren Partien unseres Irissektors um die Mittellinie herum seben wir dagegen die Dilatatorleisten häufig abgeknickt oder unregelmässig zusammengefaltet und so im ganzen der Innenfläche genähert. Daneben finden wir auch jene präformierten Hohlräume in den Leisten infolge des Zuges im Stroma in temporaler und nasaler Richtung verzogen. Sie erscheinen nicht mehr röhrenförmig, sondern mehr unregelmässig abgeplattet.

Das Studium der Flachschnitte bestätigt uns das, was wir durch Radiär- und Tangentialschnitte erkannt haben. Eine Erscheinung sei hier besonders erwähnt. Sie betrifft jene Verbindungsfasern zwischen Dilatator und Sphinkter, die vom Dilatator abzweigen und bei Mydriasis in kurzen Bogen in den Sphinkter einstrahlen. In den entsprechenden Flachschnitten bei enger Pupille verlaufen diese Fasern nicht mehr bogenförmig; sie sind hier ganz in den cirkulären Verlauf der Sphinkterfasern hineingezogen und bilden mit den radiärverlaufenden Dilatatorfasern einen stumpfen Winkel, der manchmal einem rechten nahe kommt.

\section{Unterer (ventraler) Iristeil.}

I. Bei weiter Pupille.

In der unteren Langseite der Iris finden wir einen Sektor, dex ganz analog dem eben beschriebenen oberen gebaut ist. Das Ver- 
hältnis zwischen Scheiniris und funktioneller Iris ist hier im ventralen Quadranten etwa das gleiche wie dorsal. Die vorhandenen Unterschiede sind rein gradueller Natur, wie dies aus den Erörterungen des anatomisch-physiologischen Teiles der Arbeit und aus der beigegebenen Figur (Taf. X, Fig. 11) leicht geschlossen werden kann. Die Bewegung des unteren Teiles der Iris ist nicht ganz so ausgiebig wie oben. Die anatomischen Einrichtungen entsprechen natürlich diesem Minus an Dehnung und Zusammenziehung.

So finden wir die Menge an dehnungsfähigem Stroma vermindert. Die Anhäufung in der ciliaren Hälfte ist nicht so bedeutend wie oben (Taf. X, Fig. 4). Der Sphinkter erscheint im Radiärschnitt bei Mydriasis namentlich in seiner ciliaren Hälfte nicht so stark wellenförmig gerafft. Die Bildungen an den Innenschichten der Iris, die zur Dilatatorverstärkung dienen, sind nicht so ausgeprägt. Die Strukturfalten sind weniger tief und weniger zahlreich. Auch die Dilatatorleisten sind an Zahl und Stärke verringert. Die kleineren Buckel und Vorwölbungen, knospenartigen Auswïchse und Verzweigungen sind an den Dilatatorleisten und auch sonst an der Dilatatorlamelle in nur geringer Ausbildung und Zahl vorhanden.

Bezüglich der Verbindung zwischen Sphinkter und Dilatator möge noch erwähnt werden, dass man ausser den hinter dem Sphinkter in schräg radiärer Richtung ziehenden Verbindungsfasern auch manchmal schon einen entsprechenden Radiärzug vom ciliaren Randbündel des Sphinkters nach dem Dilatator ziehen sieht. Dieses Bündel könnte man als Andeutung einer "Speichenfaser" ansprechen.

Der untere Irissektor, in dem wir ganz die analogen Verhältnisse wie in dem oberen finden, ist nicht ganz so breit als dort; er nimmt etwa nur 1/5 des ganzen Irisringes ein (Taf. IX, - Fig. 1).

Alles übrige ergibt sich ohne weiteres durch Analogieschliisse unter Berücksichtigung der graduellen Verminderung und aus den betreffenden Zeichnungen (Taf. IX, Fig. 1 u. Taf. X, Fig. 2-5).

\section{Bei enger Pupille.}

Die Ausbreitung der Iris erfolgt in der gleichen Weise wie im oberen Quadranten. Die Radiärbreite der funktionellen Iris ist hier bei Miosis nur ungefähr ${ }^{13} /{ }_{4}$ mal grösser als bei Mydriasis (Taf. X, Fig. 5). Das Studium der mikroskopischen Schnittserien bestätigt alle Befunde, die wir bereits im oberen Teile der Iris gemacht haben. Ich kann daher auf die dortigen Ausführungen verweisen, doch möchte ich es nicht verabsäumen, noch einmal hier besonders zu erwähnen, 
dass jene Formveränderungen an den Dilatatorleisten, die in Abknickung und Zusammenfaltung in den mittleren Teilen und in den seitlichen Partien im Umlegen nach der Mitte zu bestanden, auch hier ventral in gleicher Weise zu finden sind.

\section{Temporaler Iristeil.}

I. Bei weiter Pupille.

Sehen wir uns zunächst wieder einen Radiärschnitt aus der Mitte dieser seitlichen Partie an (Taf. X, Fig. 6). Sogleich fällt in die Augen, dass die Radiärbreite des ruhenden Teiles der Iris etwa gleich gross ist wie die der funktionellen Iris. Hier nimmt also die funktionelle Iris nur die Hälfte der Schein- oder Ausseniris ein. Die Entfernung zwischen den Scheiteln des Corneawinkels und denen des Irisciliarfortsatzwinkels beträgt $2-2{ }^{1} / 4 \mathrm{~mm}$, im Gegensatz zu dorsal und rentral, wo sie nur etwa $1 / 6 \mathrm{~mm}$ war. Also bildet hier der ruhende Teil der Aussen- oder Scheiniris eine breite Zone, wie dies aus der beigegebenen Zeichnung (Taf. IX, Fig. 1) ohne weiteres ersichtlich ist. Weiter sieht man, dass die ganze Breite der funktionellen Iris hier im mittleren Teil des temporalen Quadranten etwa nur $2 / 3$ der entsprechenden Irisbreite im dorsalen und ventralen Quadranten bei Mydriasis ist. Die Querschnitte der grossen Gefässe und Nerven, die wir dorsal und ventral in der ciliaren Hälfte der funktionellen Tris liegen sahen, befinden sich hier ein beträchtliches Stück peripher vom Irisciliarfortsatzwinkel im ruhenden Teil der Iris.. Die Basis am Ciliarfortsatziriswinkel ist die breiteste Stelle. Von da an verschmälert sich die Iris allmählich pupillar, um am Sehlochrande in eine stumpfe Spitze auszulaufen. Die Konturen der Aussenund Innenfläche verlaufen in ziemlich gerader Richtung und sind glatt ohne nennenswerte Einkerbung und Wölbung. Die Stromamenge ist nur gering und tritt gegen den muskulösen Apparat sichtlich zurück. Der Sphinkter nimmt etwa $2 \%_{3}$ der Breite der funktionellen Iris ein. Das Band, das die Querschnitte seiner eng aneinanderliegenden Muskelbündel bildet, liegt gestreckt in gerader radiärer Richtung. Seine grösste Breite findet sich etwas pupillar von seiner Mitte. Von da an bis zum Pupillarrande füllt er die ganze Irisdicke aus. In seinem ciliaren Teile bleibt anssen und innen ein keilförmiger Stromastreifen übrig. An der Innenseite sehen wir seine Muskelbündel nicht mehr rein quer, sondern schräg oder in der Längsrichtung getroffen. Diese mehr radiär verlaufenden Muskelfasern setzen sich mit dem direkt an der Innenfläche liegenden 
Dilatator selbst oder mit den ihm von diesem entgegenkommenden radiären Fasern in Verbindung. Nach dem ciliaren Rande zu wird das Sphinlterband schmaler, die Fasern des ciliaren Randbündels sehen wir in manchen Schnitten auch ganz oder teilweise in schräg radiärer Richtung getroffen. Sie gehen also in die Verlaufsrichtung der ihnen dort entgegenkommenden radiären, im Stroma liegenden Dilatatorfasern iber. Wir werden dieses Verhalten in den Flachschnitten bestätigt finden.

Den Dilatator sehen wir im temporalen Quadranten doppelt angeordnet. Einmal den eigentlichen am Pigment gelegenen Dilatator gebildet von den Zellen der äusseren Lage der Pars iridica retinae. Daneben finden wir aber pigmentierte Muskelfasern zu dünneren oder dickeren Bündeln angeordnet im Stroma selbst in kurzer Entfernung von der Innenfläche parallel zu ihr in radiärer Richtung nach dem Sphinkterrande hin verlaufen. Diese pigmenthaltigen Muskelfasern mit stäbchenförmigen Kernen scheinen manchmal von der randständigen Dilatatorschicht vollkommen isoliert im Stroma eingelagert zu sein. Häufig kann man aber einen lockeren Zusammenhang mit der auch schon eine teilweise Auffaserung nach dem Stroma hin aufweisenden Randschicht (Bruchsche Membran) konstatieren (Taf. X, Fig. 11). Nicht alle diese Fasern haben eine radiäre Verlaufsrichtung parallel zur Innenflächenkontur, Manche strahlen von der InnenHäche schräg radiär und pupillar in das Stroma hinein. Diese von der Randschicht abgesprengt im Stroma verlaufenden Muskelfaserbündelchen legen sich auch nicht zu Muskelstreifen oder Lagen zusammen. Sie sind vielmehr in radiärer Ausdehnung wie auch seitlich durch sich einschiebende Stromastreifen unterbrochen. Man bekommt den Eindruck, als wären von der randständigen Dilatatorschicht aus an den verschiedensten Stellen einzelne oder Gruppen ron Muskelfasern in das Stroma in schräg radiärer Richtung hineingewachsen. In der Nähe des Ciliarfortsatziriswinkels sind diese im Stroma eingelagerten Muskelfasern noch spärlich vorhanden und liegen in näcbster Nähe der Innenfläche. Pexipher von dem Winkel habe ich niemals solche Muskelfasern finden können. Pupillar werden sie immer häufiger und bekleiden in immer breiterer Zone die Innenflächenkontur. Am ciliaren Sphinkterrande und kurz vorher reichen sie am weitesten ins Stroma hinein. Sie strahlen teils direlst in das Randbündel des Sphinkters aus, dessen Fasern auch schon, wie wir oben sahen, nicht mehr rein cirkulär verlaufen, teils umfassen sie den Rand des Sphinkters. Ein grosser Teil setzt sich hinter dem Sphinkter- 
rande in dem Stromastreifen zwischen diesem und dem Pigment fort und strahlt successive in die Innenfläche des Sphinkters aus. Einheitliche auf längere Strecken verfolgbare Streifen, wie wir sie als längsgetroffene Dilatatorleisten im oberen und unteren Quadranten sahen, sind hier nicht zu bemerken. Ebensowenig finden wir hier auf den Radiärschnitten Andeutungen von ausgeprägten Strukturfalten. Dies Verhalten gilt auch von der Innenflächenkontur im Bereich des Sphinkters. Diese ist auch glatt ohne Strukturfalten. Dagegen finden sich auch hier allenthalben zwischen Dilatator und Sphinkter Verbindungsfasern im Stroma, die teils rein radiär, teils mehr oder weniger schräg oder quer vom Schnitt getroffen sind. Die rein radiär verlaufenden Fasern legen sich in der Regel mit den ihnen von der Innenfläche des Sphinkters entgegenkommenden radiären Muskelbündeln zusammen, wie bereits oben beim Sphinkter erwähnt. Betrachten wir die Serien von Tangentialschnitten aus diesem temporalen Iristeile, so finden wir in der Nähe des Ciliarfortsatziriswinkels die innere Profillinie nur wenig und flach gewellt, selten eingekerbt. Diese Unregelmässigkeit verliert sich aber bald in den folgenden Schnitten nach dem Sphinkter hin. Die Profillinie wird dann glatt und gerade. Wir finden hier also keine ausgeprägten Strukturfalten. An der Dilatatorlamelle sieht man auch keine solchen Einfaltungen, wie wir sie im dorsalen und ventralen Quadranten fanden. Es macht sich hier schon ganz in der Nähe des Ciliarfortsatziriswinkels an der Dilatatorlamelle eine Auffaserung und Ausstrablung dieser Schicht nach dem Stroma hin geltend. Einzelne oder kleine Komplexe von Zellen springen in das Stroma vor und verlieren bald einen eigentlichen Zusammenhang mit der randständigen Dilatatorschicht. Waren jene Dilatatorleisten in dem oberen und unteren Quadranten eine Einfaltung der ganzen zusammenhängenden Epithelmuskellamelle, so ist es hier mehr ein Hineinwachsen einzelner oder einer kleineren Anzahl von Zellen in das Stroma. Die einzelnen Einwüchse sind im Tangentialschnitt meist mehr oder weniger kolbenartig gestaltet (Taf. X, Fig. 11). Im verdickten Teile des Kolbens findet sich das Protoplasma mit Pigment und Kernen und zwar nach innen gelagert, während in der Peripherie die kontraktilen Elemente der Zelle liegen. Meist sind die Kolben durch einen schmalen muskulösen Streifen, der häufig einige Pigmentkörnchen enthält, stielartig mit dem randständigen auch etwas aufgefaserten Teil der Bruchschen Membran verbunden (Taf. X, Fig. 11). Manchmal aber liegen diese Zellen ganz abgesprengt scheinbar ohne Zusammen- 
hang mit dem übrigen Dilatator im Stroma. Auch hier sehen wir häufig jene Hohlräume in den Zellkomplexen auftreten, die wir als eine Begleiterscheinung der äusseren retinalen Zellschicht fanden. Diese im Stroma liegenden Zellanhäufungen nehmen dann Ringform an als Querschnitt einer kurzen Röhre. Neben solchen Röhren, bei denen nach innen das Protoplasma mit Pigment und peripher die kontraktilen Teile gelegen sind, bemerkt man auch nicht selten abgesprengte Zellkomplexe, in denen diese Anordnung nicht vorhanden zu sein scheint, vielmehr die einzelnen Elemente regellos durcheinander liegen, wie Klinge (8) es für Ziege und Schaf bei Mydriasis als Regel aufstellt. Die einzelnen Zellen selbst haben mehr den Charakter der gewöhnlichen glatten Muskelzellen, nur dass hier der Pigmentgehalt dazukommt. Dem entspricht auch die Form des Kernes, der, wie man deutlich auf den Radiärschnitten sieht, stäbchenförmig wie bei den Sphinkterfasern ist. Übrigens scheinen auch in den Muskelfasern des Sphinkters vereinzelte Pigmentkörnchen enthalten zu sein. Ich kann daher Heerfordt (7) nicht beistimmen, der annimmt, dass dieses Pigment innerhalb des Sphinkters $z$ wischen den Muskelfasern liegt.

Jene kolbenartigen Einwüchse stehen manchmal büschelartig beieinander. Sie können so dicht stehen, dass es auf dem Tangentialschnitt aussieht, als ob die inneren Trisschichten von einer mehr oder weniger kontinuierlichen Hecke solcher Dilatatorauswïchse begleitet wäre. Es finden sich dann zwischen den einzelnen Kolben und sonstigen Anhäufungen der Dilatatorzellen nur schmale Bindegewebsstränge des Stromas. Hier wird man an die Schilderung des Dilatators beim Delphin erinnert, die Grynfeltt (5) gibt. Grynfeltt beschreibt jedoch die dicke Schicht als dicht nebeneinandergereihte Falten der Dilatatorlamelle (ähnlich wie dorsal). Doch ist wohl zwischen dieser Art der Einfaltung und dem Hineinwachsen, wie wir es hier an der Dilatatorlamelle finden, ein prinzipieller Unterschied nicht zu formulieren. Sollen doch auch bei den Aktinien vollkommen von der äusseren Zellschicht abgelöste Muskelzellen in der Körperwand sich finden. Die einzelnen dieser Auswüchse kann man nicht als kontinuierliche Leisten bis nach dem Sphinkter hin verfolgen. Bei Verfolgung einzelner in der Tangentialschnittserie hören sie bald auf, und es treten andere an ihre Stelle. Wir finden also durch die Tangentialschnitte unsere Annahme, auf die uns die Radiärschnitte führten, bestätigt, dass nämlich die im Stroma liegenden radiären Muskelbündel durch Ausstrahlung von immer neuen Stellen der 
Der muskulöse Apparat der Iris des Schafes und seine Beziehungen usw. 437

randständigen Dilatatorschicht ergänzt werden. In den Tangentialschnitten aus der Nähe des ciliaren Sphinkterrandes sehen wir, dass ausser den in mehr radiärer Richtung in den Sphinkter übergehenden Verbindungsfasern noch kleinere, dünnere Verbindungsfasern von den abgesprengten Dilatatorfasern in scharfem Bogen in die cirkuläre Sphinkterfaserrichtung einbiegen, wie wir es auch im oberen und unteren Quadranten sahen. Solche scharf abbiegende Verbindungsfasern können wir auch noch hinter dem Sphinkter weiter pupillenwärts verfolgen bis gegen Ende des Dilatators hin.

Die Flachschnitte zeigen uns besonders deutlich die starke Verbindung, die in diesem Irissektor am Sphinkterrande zwischen diesem und dem Dilatator statt hat. Man sieht hier, wie sich breite Muskelbündel vom Dilatator zum Sphinkter zu einem flachen Bogen zusammenlegen. Eine Grenze zwischen Sphinkter und Dilatator ist in ihnen schwer zu ziehen. Stäbchenförmige Kerne weisen alle Fasern auf, sowohl die vom Dilatator als auch die vom Sphinkter. Auch der Pigmentgehalt ist nicht entscheidend, da derselbe innerhalb dieser Verbindungsbögen in den Zellen nach dem Sphinkter hin allmählich abnimmt. Diese Bögen finden wir auch noch weiter pupillarwärts von der Randzone des Sphinkters auftreten, wie wir dies bereits in den Radiärschnitten sahen. Neben diesen starken Verbindungsbögen sehen wir hier in den Flachschnitten auch jene kleineren kurzen Verbindungsfasern, die vom Dilatator in scharfem Bogen in die cirkulär angeordneten Sphinkterbündel übergehen. Die Flachschnitte ergänzen also jenen Befund an den Tangentialschnitten.

Der seitliche Übergang in den oberen und unteren Quadranten ist ein allmählicher. Hier wird die Radiärbreite der funktionellen Iris grösser, das Stroma nimmt an Menge zu, der Sphinkter beginnt sich im Radiärschnitt in schwache Falten zu legen, die im Stroma liegenden Dilatatorbündel nehmen an Menge ab, es treten langsam mehr Strukturfalten auf; kurz die Tris wird immer geeigneter, sich auszubreiten und zusammenzuziehen. Hier temporal finden wir oben und unten eine verhältnismässig breite $Z_{0}$ e, wo ausser ausgeprägten Strukturfalten und deutlichen Dilatatorleisten auch schon jene im Stroma eingelagerten Dilatatorbündel nebeneinander vorkommen.

\section{Bei enger Pupille.}

Betrachten wir einen Irisschnitt, der direkt an dem temporalen Winkel der bei Miosis spaltförmigen Pupille gelegen ist (Taf. X, Fig. 7), so müssen wir sagen, dass eine zu berücksich- 
tigende Formveränderung nicht qu konstatieren ist. Wir könmen diese Partie als relativ fix betrachten. Dementsprechend finden wir auch keine feineren anatomischen Veränderungen bei Miosis. Durch die Kontraltion des Sphinkters erscheint bei Miosis sein Querschnitt etwas breiter und der Randteil der Iris auf dem Radiärschnitt etwas kolbig verdickt, nicht mehr so keilförmig spitz auslaufend wie bei Mydriasis. Natürlich macht sich in den weiter dorsal und ventral gelegenen Übergangsteilen des temporalen Irisquadranten eine entsprechende Dehnung und Ausbreitung der Iris mit Streckung des Sphinkterbandes im Radiärschnitt, eine Anspannung und Verschmälerung der Dilatatorfasern usw. geltend, wie dies aus dem früher Gesagten ohne weiteres geschlossen werden kann.

\section{Nasaler Iristeil.}

\section{Bei weiter Pupille.}

Der nasale Quadrant steht zum temporalen in ähnlichem Verhältnis wie der dorsale zum ventralen, nur ist der graduelle Abstand noch etwas vergrössert. Auch hier ist überall eine graduelle Verminderung der Eigenschaften im Aufbau gegenüber dem temporalen Quadranten vorhanden. Dazu kommt, dass sich in den weiter dorsal und ventral gelegenen Teilen ein Überspielen der stark graduell verminderten Verhältnisse des dorsalen und ventralen Quadranten deutlich bemerkbar macht, was ja mit der Abschwächung jener für den temporalen Quadranten charakteristischen Eigenschaften Hand in Hand gehen muss. Um weitschweifige Wiederholungen zu vermeiden, will ich nur einige markante Unterschiede herausgreifen, aus denen sich unter Berücksichtigung meiner früheren eingehenderen Ausführungen die Einzelheiten ohne weiteres ergeben. Wenn wir die Radiärschnitte betrachten (Taf. $\mathbf{X}$, Fig. 8), so finden wir, dass der ruhende Teil der Iris hier nasal, etwa $1-1 y_{4} \mathrm{~mm}$ breit ist, also etwa halb so breit wie temporal, doch noch beträchtlich breiter als dorsal und ventral. Die Radiärbreite der funktionellen Tris bei Mydriasis ist nasal etwa die gleiche wie temporal in beiden Stadien. Die Querschnitte der grösseren Gefässstämme und Nerven fallen in die Grenzgegend der ruhenden und funktionellen Iris, zum Teil schon in den peripheren Teil der letateren. Die Stromamenge ist infolgedessen im funktionellen Teil der Iris auch eine grössere als temporal, doch ist die Form der Iris im ganzen noch gestreckt und keilförmig; allerdings ist sie an der Stelle, wo die Gefässe liegen, etwas verdickt.

Das Sphinkterband liegt auch gestreckt, doch erscheinen die 
Bündel nicht so dicht gehäuft und sind kleiner und spärlicher als temporal. Von seiner Innenfläche gehen auch Bündel in schräg radiärer Richtung zum Dilatator, von dem eben solche schwache Fasern ihm entgegenkommen, ganz wie temporal, nur graduell vermindert. Der Dilatator besteht auch aus der etwas aufgefaserten randständigen Schicht und aus im Stroma liegenden schwachen Radiärbündeln. Dieselben reichen aber nicht so weit in das Stroma hinein. Relativ am stärksten sind sie aber auch entwickelt am ciliaren Sphinkterrande und kurz davor. Die Verbindung zwischen Sphinkter und Dilatator bildet die Analogie zu dem temporalen Quadranten.

In den Serien von Tangentialschnitten sehen wir die Dilatatorschicht genau so aufgefasert und in derselben Form Einwüchse in das Stroma hineinschicken. Die Einwüchse sind nur bedeutend spärlicher und kleiner als in den entsprechenden Teilen des temporalen Quadranten. Sie reichen auch nicht so weit in das Stroma hinein, wie bereits oben erwähnt. Die Andeutungen von Strukturfalten in der ciliaren Zone sind ausgeprägter, namentlich nach den dorsalen und ventralen Übergangsschenkeln zu. Hier finden wir auch manchmal an der randständigen Schicht der Dilatatorlamelle kleine kurze Leisten, die grosse Ähnlichkeit mit den Leisten haben, die Klinge (8) beim Schwein fand.

Der Übergang von der oberen und unteren Irispartie nach der nasalen Seite hin geht viel allmählicher vor sich als der nach der temporalen Seite. Wir finden hier nasal je eine Zone, wo wir nur eine randständige Dilatatorschicht mit mässigen Strukturfalten auf dem Tangentialschnitte konstatieren können. Erst langsam gesellen sich hierzu nach dem nasalen Pupillarwinkel hin geringe Auffaserung und kleine spärliche Einwüchse ins Stroma. Von den Flachschnitten sei noch erwähnt, dass sich auch hier jene flachen Verbindungsbögen zwischen Dilatator und Sphinkter vorfinden, nur sind sie schwächer.

\section{Bei enger Pupille.}

Im Gegensatz zu temporal vermag man im nasalen Quadranten bei Miosis schon eine merkliche Formveränderung zu konstatieren, doch ist sie noch klein im Vergleich mit den entsprechenden Veränderungen im dorsalen und ventralen Quadranten. Auf dem Radiärschnitte sieht man, wie die ganze funktionelle Iris gestreckter ist (Taf. X, Fig. 9). Thre Radiärbreite ist etwa um 1/2 grösser geworden, als sie bei Mydriasis war. Die Dicke des sphinkterfreien Teiles der funktionellen Iris hat auch etwas abgenommen. Der Sphinkterv. Graefe's Archiv für Ophthalmologie, LXX. 3. 
querschnitt ist infolge der Kontraktion verbreitert, so dass die Randpartie des radiären Querschnittes der Iris wie temporal geringgradig kolbig verdickt erscheint. Am Dilatator kann man hier nasal auch schon eine Dehnung seiner einzelnen Elemente konstatieren. Unter anderem tritt die Auffaserung an den kontraktilen Teilen der äusseren Retinalamelle nach dem Stroma und den in diesen eingelagerten Muskelfasern nicht mehr so deutlich hervor. Die Kontur der Bruchschen Membran ist glatter. Die vorhandenen Strukturfalten sind bis auf geringe Andeutungen in der Nähe des Ciliarfortsatziriswinkels verstrichen, ebenso die kleinen Dilatatorleisten.

Whe ich an die Zusammenfassung der Resultate meiner Arbeit gehe, muss ich noch einige Fragen erörtern, bei denen meine Befunde mit den Ansichten von Autoren, die auf gleichem Gebiete gearbeitet haben, nicht ohne weiteres in Einklang zu bringen sind.

Die Ergebnisse der Arbeit Klinges (8) bezüglich des Schafes habe ich durch meine Untersuchungen zum grossen Teil bestätigen können, namentlich was den feineren Aufbau des Dilatators anbetrifft. Seine abweichenden Befunde erklären sich leicht daraus, dass er den Irisring in seiner ganzen Circumferenz auch bei den Tieren mit querovaler Pupille als einheitlich aufgebaut angenommen hat und daraus, dass ihm keine durchgehenden Serien zur Verfügung standen, wie ich dies schon eingangs erwähnte. So sah Klinge, dass die Dilatatorleisten in sogenannte Speichenfasern am ciliaren Sphinkterrande übergingen und verallgemeinerte diese Ansicht. Nach meinen Untersuchungen muss ich diesen Befund dahin richtig stellen, dass ich im mittleren Teil des dorsalen und ventralen Quadranten die Dilatatorleisten immer ciliar vom peripheren Sphinkterrande aufhören oder in Strukturfalten übergehen sah. Solche Speichenfasern, die im sphinkterfreien Teil von der inneren Epithellamelle zum ciliaren Sphinkterrande verlaufen, sah ich in dem dorsalen Sektor niemals, dagegen manchmal im entsprechenden ventralen. Durch diese letzteren kann man, wenn nur Radiärschnitte zur Verfügung stehen und keine Serien von Tangentialschnitten, leicht zur Annahme verleitet werden, dass die Dilatatorleisten im ventralen mittleren Irissektor in diese Speichenfasern übergehen. Noch leichter muss man zu dieser Annahme kommen, wenn man Schnitte aus den Übergangszonen nach den Seiten hin, namentlich nach temporal vor sich hat; denn hier sind neben jenen echten Dilatatoreinfaltungen, die für dorsal und 
ventral typisch sind, auch schon jene ins Stroma eingelagerten losgesprengten Dilatatormuskelfasern vorhanden, die wir temporal und nasal immer in die Randbündel des Sphinkters einstrahlen sahen.

Jene Form der Dilatatorleisten, wie sie Klinge beim Schaf bei Mydriasis schildert, fand ich namentlich in der Übergangszone des ventralen und dorsalen in den nasalen Quadranten und auch sonst in der Nähe des Ciliarfortsatziriswinkels.

Eine solche Umwandlung in Röhren bei Miosis, wie sie Klinge schildert, konnte ich jedoch an ihnen nicht feststellen. Ich sah jene Leisten bei Miosis meist ganz verschwinden. Klinges Befunde erkläre ich mir daraus, dass er bei Miosis vielleicht Schnitte aus einer anderen Partie des Irisringes vor sich hatte und dass in dieser Iris gerade jene Hohlräume präformiert waren, die ich als eine Sekretionswirkung und temporäre Erscheinung an der Dilatatorzellschicht ansprechen möchte.

Bezüglich des Vorkommens von ausgeprägten tiefen Dilatatorfalten beim Schaf hat meine Arbeit die darauf bezüglichen Befunde Grynfeltts (5) voll und ganz bestätigt. Ich muss daher der Ansicht Heerfordts in der Nachschrift zu seiner Arbeit (7), dass eine Verwechslung der Dilatatorleisten mit radiären Sphinkterbündeln nicht auszuschliessen sei, entgegentreten. Ich glaube vielmehr, dass meine Befunde beim Schafe dazu angetan sind, eine vermittelnde und ergänzende Rolle zu spielen. Heerfordt formuliert einen scharfen Unterschied zwischen glatten Muskelzellen des Sphinkters und den Epithelmuskelzellen der Dilatatorlamellen und zählt Unterscheidungszeichen auf, die nach seiner Ansicht zwischen radiären Sphinkterbündeln und Dilatatorfalten aufgestellt werden können. Ich halte nun dafür, dass jene ins Stroma ,eingewachsenen" und eingewanderten. muskulösen Fasern der Dilatatorschicht, die ich beim Schaf im temporalen und nasalen Quadranten fand, eine Art Übergangsform darstellen zwischen den Muskelzellen des Sphinkters und den Epithelmuskelzellen, die sich in der äusseren Retinaschicht mit ihren echten Einfaltungen vorfinden. Indem die einzelnen Muskelepithelzellen der Dilatatormembran in das Stroma hineingewandert und immer mehr einen eigentlichen Zusammenhang mit ibrem Mutterboden der äusseren Retinalamelle verloren haben und isoliert im Stroma liegen, haben sie auch mehr und mehr ihren ursprünglichen Charakter als Epithelmuskelzellen verloren. Die kontraktilen Elemente haben auf Kosten des protoplasmatischen Teiles der Zelle zugenommen, der Rest dieses letzteren samt dem Kerne ist in die spindelförmige Muskelzelle hin- 
eingezogen, die Zelle hat ganz den Charakter einer gewöhnlichen glatten Muskelzelle mit stäbehenförmigem Kerne angenommen, nur mit dem Unterschiede, dass sie Pigment enthält. Heerfordt selbst bebt in seiner Nachschrift hervor, dass er „an Radiärschnitten der Kanincheniris hie und da vor der Dilatatorschicht radiäre Bündel mit ausgeprägten stabförmigen Kernen gesehen habe"s. Nun habe ich in meiner Arbeit gezeigt, dass beim Schaf diese Dilatatoreinwüchse, die aus glatten Muskelzellen mit stäbchenförmigen Kernen und Pigmentgehalt zusammengesetzt sind, allenthalben in den Sphinkter ausstrahlen, und dass in den Verbindungsbögen zwischen Sphinkter und Dilatator nicht entschieden werden kann, wo Dilatatorfasern aufhören und Sphinkterfasern beginnen; denn auch die glatten Muskelzellen, die schon eine ganz ausgeprägte cirkuläre Anordnung aufweisen, zeigen, namentlich wenn sie in der Nachbarschaft der Verbindungsfasern liegen, noch deutlichen Pigmentgehalt. Vereinzelte Pigmentkörnchen findet man auch nicht selten in den Zellen des Sphinkters überhaupt. Also lässt sich auch nicht in Ruicksicht auf das Pigment entscheiden, wo die Grenze zwischen Sphinkter und Dilatator zu ziehen ist. Die Frage, was ist Sphinkter, was Dilatator, dürfte sich vielleicht überhaupt nur in Rücksicht auf die Faserverlaufsrichtung und die Innervation einigermassen entscheiden lassen. Aber auch dann dürfte man noch auf $Z_{\text {weifel stossen, wenn man } z u}$ entscheiden hätte, welchem Muskelsystem man die einzelnen Verbindungsfasern zurechnen sollte.

Diese Deutung meiner Befunde steht nicht im Gegensatz zu der für die Dilatatorfrage so entscheidend gewordenen Ansicht Heerfordts, die Zellen der äusseren retinalen Lamelle als Epithelmuskelzellen anzusprechen, ähnlich den in der Wand von Aktinien. Ich glaube vielmehr, dass meine Befunde eine Analogie bilden zu der Tatsache, dass auch in der Wand der Aktinien von der ektodermalen äusseren Zellage losgesprengte Muskelzellen zu finden sind.

Hierbei möchte ich noch auf jene Hohlraumbildung aufmerksam machen, die wir an die Dilatatorlamelle gebunden fanden und die ich mir als eine Art Sekretion zu erklären suche. Wenn man annimmt, dass jene Epithelmuskelzellen der äusseren Retinalamelle noch eine sekretorische Tätigkeit entfalteten, so würde dies ihrer ektodermalen Abstammung keineswegs zuwiderlaufen. Finden wir doch auch an den Epithelzellen des Traubenkornes, die gleichfalls der Pars iridica retinae angehören, Veränderungen, die auf eine sekretorische Tätigkeit der Zellen hindeuten, wie es Zietzschmann(12) 
Der muskulöse Apparat der Iris des Schafes und seine Beziehungen usw. 443

in seiner Arbeit über die Traubenkörner unserer Haustiere nachgewiesen hat. Dies ist noch ein Grund mehr, auch jerte Hohlräume in den Dilatatorleisten, die Klinge als eine Folgeerscheinung der Dehnung der Iris bei Miosis anspricht, als Sekretionsvorgang zu erklären.

Im physiologisch-anatomischen Teil meiner Arbeit bei den Ausführungen, durch die ich die Mechanik der Irisbewegung beim Schafe zu erklären versuchte, habe ich dem Stroma eine relativ passive Rolle gegenuiber dem Muskelapparat zuerteilt.

Nun kommt Münch(9) bei seinen eingehenden und interessanten Studien an der Iris des Menschen zu dem Schlusse, dass die Stromazellen muskulöser Natur sind und das Stroma selbst sich infolgedessen zusammen mit dem kontraktilen Teil der Epithelmuskellamelle beim Pupillenspiel aktiv beteiligen muss.

Wenn ich beim Schafe den infolge der Verstärkung verhältnismässig gut entwickelten Dilatator berücksichtige, wenn ich mir die augenfällige Übereinstimmung seiner morphologischen und graduellen Entwicklung mit den in den einzelnen Abschnitten des Irisringes ihm zufallenden Aufgaben vergegenwärtige, wenn ich weiterhin erwäge, dass man die Natur des Stromagewebes des Menschen nicht obne weiteres auf das Irisstroma des Schafes übertragen kann, da dieses bei den Haustieren überhaupt mehr den Charakter des gewöhnlichen Bindegewebes hat und anch in bezug auf die Entwicklung der Gefässmuskulatur abweicht, so glaube ich aus allem diesen schliessen zu dürfen, dass beim Schafe das Stroma selbst doch eine relativ passive Rolle spielen muss gegenüber dem eigentlichen Muskelapparat (Sphinkter und epithelialen Dilatator). Ich glaube auch, dass durch mannigfaltige Unebenheiten und Ausstrahlungen des Dilatators nach dem Stroma hin dieses mit dem kontraktilen Dilatator in innigem Zusammenhange steht. Durch diese Verbindung und durch bestimmte Faseranordnung im Stroma selbst wird daher auch die Form und Spannung des Stromas von den muskulösen Dilatatorfasern in gewissem Grade beherrscht werden können. Auch aus diesem Grunde meine ich für das Schaf zur Erklärung der Formveränderung der Iris beim Pupillenspiel die Annahme einer deutlich zutage tretenden aktiven Stromamithilfe entbehren zu können.

Zum Schlusse muss ich noch die Arbeit von Eversbusch(3): "Der anatomische Grund der spaltförmigen Pupille" eingehender besprechen, da diese von der meinigen innig berührt wird.

Eversbusch untersuchte genauer die Iris des Pferdes. Er fand 
in den Sektoren der Iris, die um die Enden der spaltförmigen Pupille gelegen sind, einen Hemmungsapparat, dem er den Namen Ligamentum inhibitorium seu triangulare iridis gibt. Dieser stellt sich nach der Schilderung von Eversbusch etwa so dar: Die cirkulären Sphinkterfasern an den Enden des pupillaren Querspaltes sind durchwoben von radiärverlaufenden Muskelfasern, die auch weiter peripher die cirkulären Sphinkterfasern durchflechten. Ebenso zweigen sich aus den peripheren Sphinkterteilen Muskelfibrillen ab. Beide Fasergruppen verjüngen sich peripher vom Sphinkter zu dünneren radiären Strängen, die etwa in der Mitte zwischen Pupillar- und Ciliarrand ihren muskulösen Charakter verlieren und in starre Endbalken von elastischer Natur übergehen. "Von da ab bis gegen den peripheren Ansatzpunkt der Regenbogenhaut lösen sie sich in gegen den Oiliarrand wieder etwas breiter werdende schmale Leisten auf, welche zusammengesetzt sind aus meist nahezu parallel zueinander verlaufenden, bisweilen sich leicht überkreuzenden starren glänzenden Balken. Nur hie und da gewahrt man noch eine Muskelzelle."

Der Dilatator stellt sich nach Eversbusch in der Iris des Pferdes dar als radiäre Abzweigungen von den äussersten Lagen des Sphinkters, die ciliarwärts geschlängelt verlaufen. In den ciliaren Randteilen sind Queranastomosen vorhanden, die jedoch keinen geschlossenen Ring bilden. In den Seitenteilen der Iris findet sich ganz die gleiche Anordnung des Dilatators, nur viel spärlicher.

Wenn ich diese Schilderung Eversbuschs sowie auch seine $\mathrm{Ab}$ bildung eines Flachschnittes aus den seitlichen Teilen der Iris des Pferdes mit meinen Befunden in den entsprechenden Irissektoren beim Schafe vergleiche, und wenn ich zugleich den damaligen Stand der Dilatatorfrage berïcksichtige, so drängt sich mir die Vermutung auf, dass beim Pferde ähnliche Verhältnisse vorhanden seien, wie ich sie beim Schaf gefunden, und dass Eversbusch jene im Stroma liegenden Dilatatorfasern und ihre innige und straffe Verbindung mit dem Sphinkter als seinen Hemmungsapparat gedeutet hat.

Seine Befunde bei Betrachtung der Iris mit blossem Auge und mit Hilfe der Lupe, wobei sich nämlich die Anwesenheit des Hemmungsapparates in diesen seitlichen Partien der Iris durch stark hervortretende radiäre Streifenbildung an der Innenfläche bemerkbar machen soll, kann ich beziuglich der Schafiris nicht bestätigen. Ich habe vielmehr an der Irisinnenfläche beim Schafe gerade in dem mittleren Teil des oberen und unteren Quadranten häufig eine deutlich radiäre Streifung konstatieren können, ein Befund, der ohne weiteres 
Der muskulöse Apparat der Iris des Sehafes und seine Beziehungen usw. 445

aus den dort stark ausgeprägten radiären Strukturfalten und Dilatatorleisten $\mathrm{zu}$ erklären ist.

In einer späteren Arbeit hoffe ich dieser Frage ähnlich wie beim Schafe auch beim Pferde näher treten zu können.

\section{Zusammenfassung.}

Die Ergebnisse meiner Untersuchungen an der Iris des Schafes möchte ich kurz in folgenden Sätzen zusammenfassen:

1. Die Iris des Schafes, so wie sie sich bei Betrachtung von der vorderen Augenkammer aus darbietet und ihre periphere Umgrenzung durch die Scheitelpunkte des Iris-Corneawinkels erhält, kann sich nicht in ihrem ganzen Umfange an der Verengerung und Erweiterung der Pupille aktiv beteiligen. Diese "Schein- oder Ausseniris" zerfällt vielmehr dadurch, dass sich die Basis der Ciliarfortsätze auf der Innenfläche der Iris pupillenwärts vorschiebt, in einen zentral gelegenen Teil, die eigentliche „funktionelle Iris", und in eine periphere Zone, den "ruhenden Teil" der Iris. Die Grenze ist ringsum in dem Scheitelpunkte des Irisciliarfortsatzwinkels zu suchen. Bis zu dieser Stelle sitzen auf der Innenfläche der Iris noch Ciliarfortsätze auf, so dass man diesen "ruhenden Teil" der Iris in gewissem Sinne noch dem Accommodationsapparate zuzählen muss. Eine Beteiligung am Pupillenspiel ist für diese periphere Zone der Iris daher auszuschliessen. Auf der andern Seite ist der Dilatator nur bis zum Scheitel des Irisciliarfortsatzwinkels hin ausgebildet. Auch aus diesem Grunde ist an dieser Stelle die periphere Grenze der „funktionellen Tris" zu suchen.

2. Die periphere Umgrenzung der "Schein- oder Ausseniris" ist eiförmig. Nun schieben sich die Ciliarfortsätze in der Gegend der Pole des Ovales, also temporal und nasal, bedeutend weiter zentralwärts auf der Innenfläche der Iris vor als dorsal und ventral. Die Breite des „ruhenden Teiles" der Iris ist also dorsal und ventral sehr gering, bedeutend grösser temporal, etwas weniger nasal. So kommt es, dass die periphere Umgrenzung der "funktionellen Iris" nahezu einen Kreis darstellt, der in das Oval der "Schein- oder Ausseniris" einbeschrieben ist.

3. In der kreisförmigen Umgrenzung der funktionellen Iris liegt nun bei Miosis der Pupillenspalt in querer Richtung, jedoch nicht symmetrisch in der Mitte, sondern etwas nach der temporalen und ventralen Seite hin verschoben. Die Radiärbreite der funktionellen Iris ist daher dorsal und rentral am grössten, ventral etwas geringer. An 
den Enden des Pupillenspaltes ist die funktionelle Iris bedeutend schmaler und zwar nasal etwas breiter als temporal.

4. Bei Mydriasis wandelt sich der miotische Querspalt der Pupille in eine querovale bis kreisförmige Öffnung um. Beim Pupillenspiel verändert sich infolgedessen die Radiärbreite der funktionellen Tris dorsal und ventral am meisten und $z$ war dorsal in stärkerem Masse als ventral. Temporal ist fast gar keine Veränderung zu konstatieren, schon in merklich höherem Masse nasal.

5. Das Ruhestadium der Iris ist bei Miosis zu suchen.

6. Am Sphinkter, der in allen Quadranten der Iris eine etwa gleichbreite pupillare Randzone einnimmt, finden sich, ausser gewissen straffen innigen Verbindungen mit dem. Dilatator in den seitlichen Irispartien, an den Enden des Pupillenspaltes keine stärker hervortretenden regionären morphologischen Verschiedenheiten.

7. Die Verschiedenartigkeit der Aufgaben beim Pupillenspiel in den einzelnen Teilen des Irisringes, deren Erfiullung also dem Dilatator zufällt, spiegelt sich in seiner verschiedenen morphologischen Entwicklung wieder:

a. Im dorsalen und ventralen Quadranten, wo die Iris beim Pupillenspiel den grössten Ausschlag macht, ist der Dilatator durch ausgiebige Faltenbildung (Strukturfalten und Dilatatorleisten) entsprechend verstärlkt und zwar ventral in minderem Grade, entsprechend dem kleineren Ausschlage in diesem Irissektor.

b. Temporal und nasal finden sich ausser der Epithelmuskellamelle an der Innenfläche der Iris vor dieser im Stroma eingelagert radiäre Muskelzüge, die Einwïchse von Zellen der Dilatatorlamelle darstellen. Diese gehen mit den Sphinkterfasern eine innige und straffe Verbindung ein. Sie dienen dazu, die seitlichen Partien des Stromas in der Nachbarschaft der Pupillenspaltenden in ihrer Form und Breite zu fixieren. Wenn also der Sphinkter nach Erschlaffung der Dilatatorfasern zur Wirkung kommt, werden diese in den Seitenteilen eingelagerten Muskelfaserzüge das dortige Stroma stiitzen und verhindern, dass auch nasal und temporal das Irisstroma gegen das Zentrum rorgezogen wird. So resultiert eine querovale bzw. spaltförmige Pupille. Da nasal dieser Hemmungsapparat in geringerem Grade ausgebildet ist, so ist es verständlich, dass dort der Pupillarm rand der Iris bei Verengerung des Sehloches einen schwachen zentripetalen Aussehlag ergibt, der temporal ganz fehlto

Vorliegende Arbeit wurde im Veterinär-anatomischen Institute der Universität Zürich unter Anleitung meines verehrten Chefs, Herm 
Der muskulöse Apparat der Iris des Schafes und seine Beziehungen usw. 447

Prof. Dr. Zietzschmann, angefertigt. Ihm auch an dieser Stelle für die Anregung zu dieser Arbeit, sowie für die allzeitig erteilten freundlichen Ratschläge und Unterstützungen meinen herzlichen Dank auszusprechen, ist mir eine angenehme Pflicht.

\section{Literaturverzeichnis.}

1) Alfieri, Alex., Un nuovo methodo per la depigmentazione dei tessuti. Monitore Zoologico Italiano. August 1897.

2) Apáthy, Aufklebemethode mit Wasser, Eiweiss, Celloidin, angegeben in A. B. Lee u. P. Mayer. Grundzüge der mikrosk. Technilk f. Zoologen u. Anatomen. 3. Aufl. 1907.

3) Eversbuseh, Vergleichende Studien über den feineren Ban der Iris. Zeitschr. f. vergleich. Augenheilk. 1882.

4) Grünhagen, Uber das Vorkommen eines. Dilatator pupillae in der Iris beim Menschen und den Säugetieren. Zeitschr. f. rat. Med. Bd. XXVIII. 1866.

5) Grynfeltt, Le muscle dilatateur de la pupille chez les mammifères. Ann. d'oculiste. Tom. LXXI. 1898.

6) Grynfeltt u. Mestresat, Remarques sur l'emploi de quelques procédés de la dépigmentation des conpes histologiques. Montpellier Médical. p. 4.

7) Heerfor d t, Studien über den Musc. dilatator pupillae. Anat. Hefte. Bd. XIV. 1900.

8) Klinge, E., Die inneren Irisschichten der Haussäugetiere. Anat. Hefte. I. Abt. Bd. XXXVI. Heft. 110. 1908.

9) Münch, Über die Mechanik der Irisbewegung. Arch. f. Ophth. Bd. LXIV. 1906.

10) Olt, Das Aufkleben mikroskopischer Schnitte. Zeitschr. f. wissenschaftl. Mikroskopie. Bd. XXIII, 3. S. 323-328 und Deutsche tierärztl. Wochenschr. 14. Jahrg. Nr. 31. 1906 .

11) Zietzschmann, Das Sehorgan. Im Handb. d. vergleich. mikr. Anatomie d. Haustiere v. Ellenberger. Berlin 1906.

12) - Die Traubenkőrner unserer Haussäugetiere. Arch. f. mikrosk. Ànat. Bd. LXV. 1905.

Erklärung der Abbildungen auf Taf. IX u. X, Fig. 1-11.

Fig. 1. Schema der Iris des Schafes bei enger und weiter Pupille.

Fig. 2-9. Radiärschnitte aus der Mitte des dorsalen (Fig. 2 u. 3), ventralen (Fig. 4 u. 5), temporalen (Fig. 6 u. 7) und nasalen (Fig. 8 u. 9) Quadranten der Iris bei Mydriasis (Fig. 2, 4, 6,8) und Miosis (Fig. 3, 5, 7, 9). Vergrösserung ungefähr 6 fach.

Fig. 10 u. 11. Tangentialschnitte aus dem temporalen (Fig. 10) und dorsalen (Fig. 11) Quadranten. 


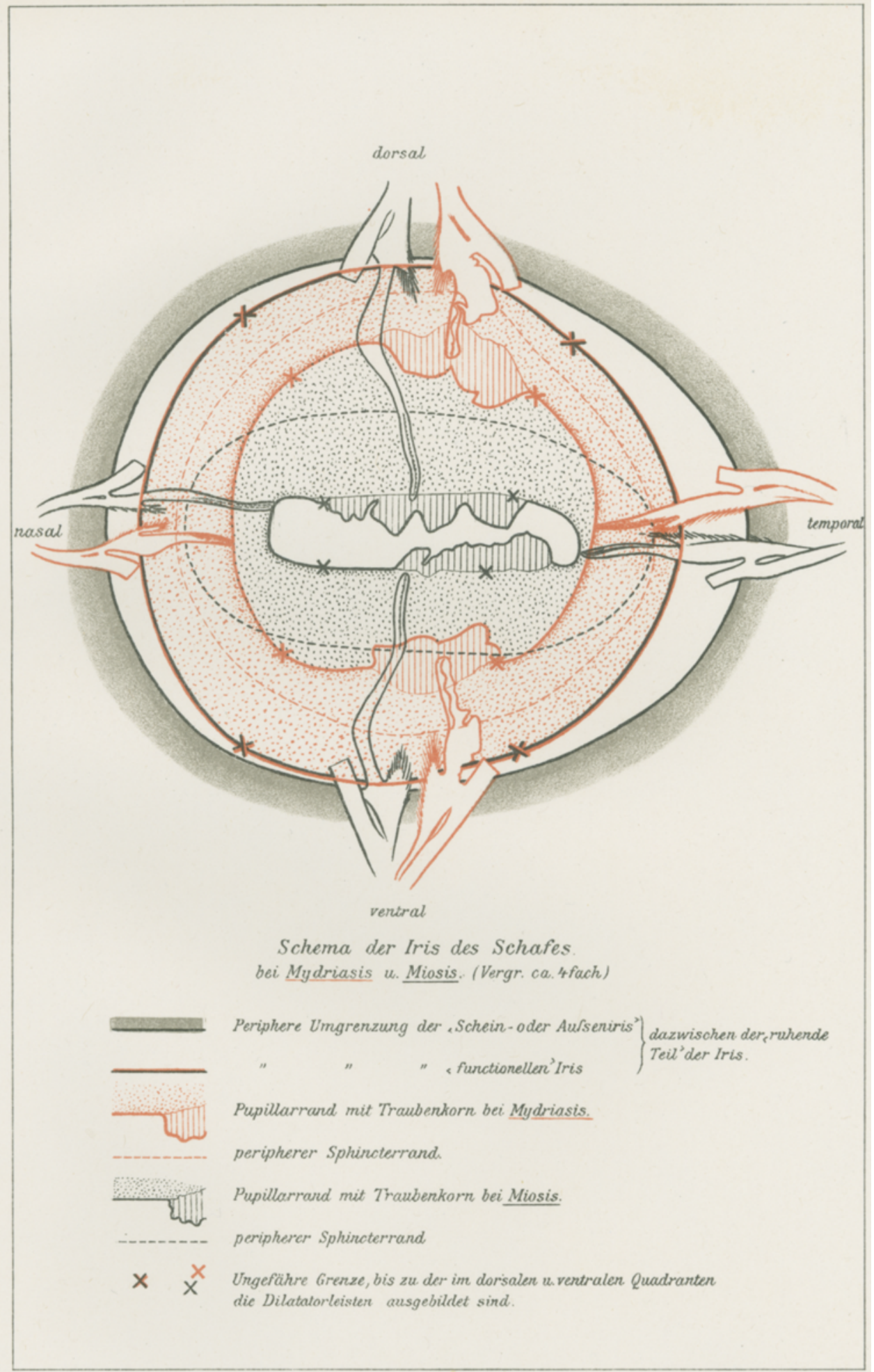




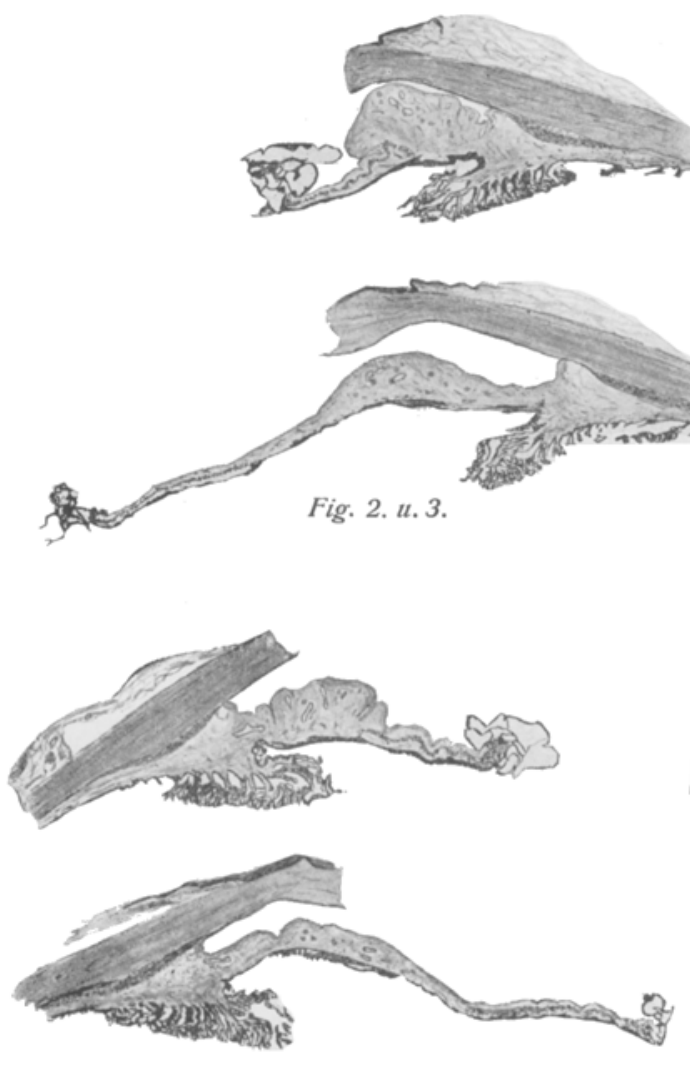

Fig. 4. u. 5.

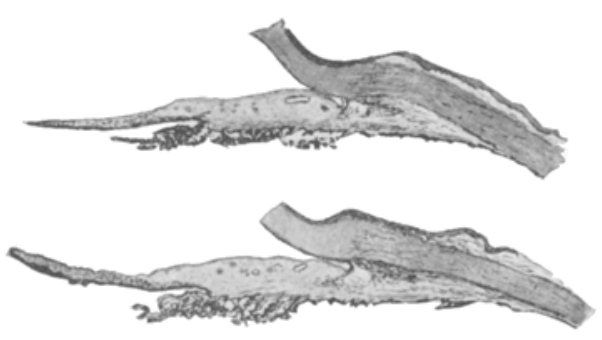

Fig. 6. u. 7.

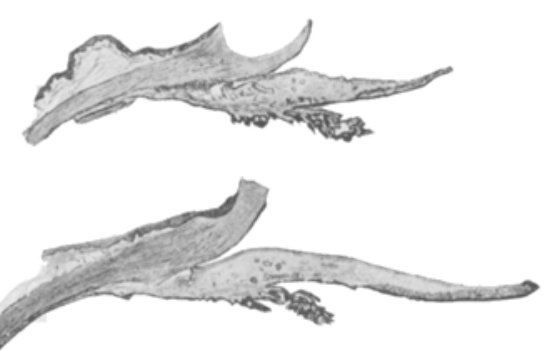

Fig. 8. u. 9.

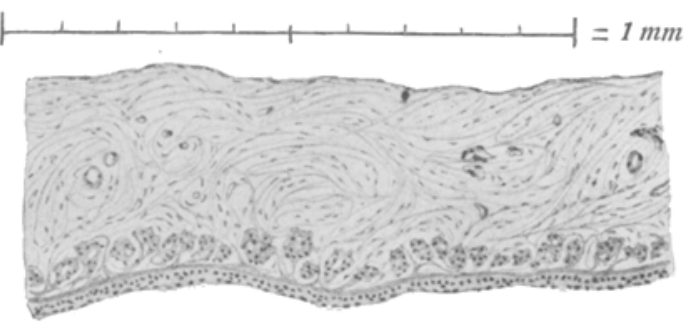

Fig. 11.

Fig. 10.
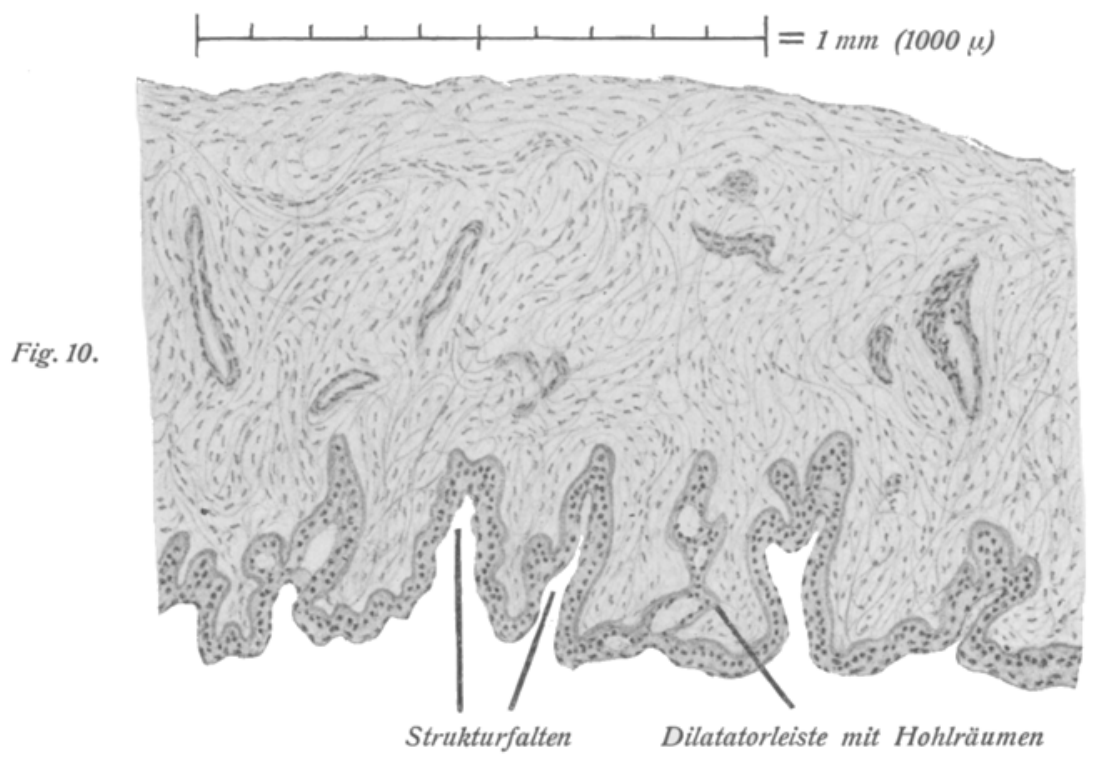

Verlag von Wilhelm Engelmann in Leipzig. 